\title{
Fourier-Mukai transforms and Bridgeland stability conditions on abelian threefolds
}

\author{
Antony Maciocia and Dulip Piyaratne
}

\begin{abstract}
We use the ideas of Bayer, Bertram, Macrí and Toda to construct a Bridgeland stability condition on a principally polarized abelian threefold $(X, L)$ with $\mathrm{NS}(X)=\mathbb{Z}[\ell]$ by establishing their Bogomolov-Gieseker-type inequality for certain tilt stable objects associated with the pair $\left(\mathcal{A}_{\sqrt{3} \ell / 2, \ell / 2}, Z_{\sqrt{3} \ell / 2, \ell / 2}\right)$ on $X$. This is done by proving the stronger result that $\mathcal{A}_{\sqrt{3} \ell / 2, \ell / 2}$ is preserved by a suitable Fourier-Mukai transform.
\end{abstract}

\section{Introduction}

In [Bri07] Bridgeland introduced the notion of stability conditions on triangulated categories. These now have many applications to the study of the geometry of the underlying spaces. Moreover, they highlight the role played by the derived categories of suitable categories of sheaves. The space of stability conditions is known precisely for curves and for abelian surfaces and Bridgeland's geometric stability conditions provide examples for all projective surfaces (see, for example, [Bri08, Mac07, AB13, Oka06]). A conjectural construction of Bridgeland stability conditions for projective threefolds was introduced in [BMT13]. The problem is reduced to proving that an inequality, which the authors call a Bogomolov-Gieseker-type (B-G-type for short) inequality, holds for certain tilt-stable objects. This inequality has been shown to hold for three-dimensional projective space (see [BMT13, Mac12]) and smooth quadric threefolds (see [Sch14]), and some progress has been made for more general threefolds (see [Tod14, LM12]). However, there is no known example of a stability condition on a projective Calabi-Yau threefold. This case is especially significant because of the interest from mathematical physics and also in connection with Donaldson-Thomas invariants. In this paper, we establish the existence of a stability condition on a particular Calabi-Yau threefold (namely, a principally polarized abelian threefold with Picard rank one). However, it is likely that the method will generalize to other Calabi-Yau threefolds. The extension to other stability conditions for the abelian threefold case will be the subject of a forthcoming article.

We reduce the requirement of the B-G-type inequality to a smaller class of tilt-stable objects as defined in Definition 2.2. These are essentially minimal objects (also called simple objects

Received 28 June 2013, accepted in final form 5 October 2014.

2010 Mathematics Subject Classification 14F05 (primary), 14J30, 14J32, 14J60, 14K99, 18E30, 18E35, 18E40 (secondary).

Keywords: Bridgeland stability conditions, Fourier-Mukai transforms, abelian threefolds, Bogomolov-Gieseker inequality.

This journal is (C) Foundation Compositio Mathematica 2015. This article is distributed with Open Access under the terms of the Creative Commons Attribution Non-Commercial License, which permits non-commercial reuse, distribution, and reproduction in any medium, provided that the original work is properly cited. For commercial re-use, please contact the Foundation Compositio Mathematica.

The second author was funded by Principal's Career Development Scholarship programme and Scottish Overseas Research Student Awards Scheme of the University of Edinburgh. 


\section{FMTS AND STABILITY CONDITIONS ON ABELIAN THREEFOLDS}

in the literature) of the heart of the stability condition. In this paper we use Fourier-Mukai theory to prove the B-G-type inequality for these minimal objects by showing that the heart is preserved by a suitable Fourier-Mukai transform (or FMT for short). For the surface case, the fact that a countable family of (Bridgeland's) geometric stability conditions satisfies the numerical conditions for being a stability condition is actually equivalent to the existence of a FourierMukai transform preserving the heart. The forward implication was proved by Huybrechts (see [Huy08]) and the reverse implication is a fairly straightforward exercise (partly done in [Yos09]). For the threefold case, we build on these ideas to establish the reverse implication.

Throughout this paper our abelian varieties will be principally polarized with Picard rank one over $\mathbb{C}$. Let $(X, L)$ be an abelian variety of dimension three and let $\ell$ be $c_{1}(L)$. We use $L$ to canonically identify $X$ with $\mathbf{P i c}^{0}(X)$. Let $\Phi: D^{b}(X) \rightarrow D^{b}(X)$ be the (classical) FMT with the Poincaré line bundle on $X \times X$ as the kernel. Then the image of the category $\operatorname{Coh}(X)$ under $\Phi$ is a subcategory of $D^{b}(X)$ with non-zero $\operatorname{Coh}(X)$-cohomology in positions $0,1,2$ and 3 . In Section 4 , we study the slope stability of $\operatorname{Coh}(X)$-cohomology under the transform $\Phi$ in great detail. In particular, we investigate the images under $\Phi$ of torsion sheaves supported in dimensions one and two, and of torsion-free sheaves whose Harder-Narasimhan-semistable (or H-N-semistable for short) factors satisfy certain slope bounds.

In [BMT13] and [BBMT14], the authors construct their conjectural stability condition hearts as a tilt of a tilt. The first tilt of $\operatorname{Coh}(X)$ associated with the H-N filtration with respect to the twisted (slope $\mu_{\omega, B}$ )-stability is denoted by $\mathcal{B}_{\omega, B}$ and the second, associated with the H-N filtration with respect to the tilt (slope $\nu_{\omega, B}$ )-stability, by $\mathcal{A}_{\omega, B}$. We shall consider the particular case where $\omega=\sqrt{3} \ell / 2$ and $B=\ell / 2$. Let $\Psi:=L \Phi$ and $\hat{\Psi}:=\Phi L^{-1}[1]$. At the end of Section 4 we prove that the images of the abelian category $\mathcal{B}_{\sqrt{3} \ell / 2, \ell / 2}$ under the Fourier-Mukai transforms $\Psi$ and $\hat{\Psi}$ have non-zero $\mathcal{B}_{\sqrt{3} \ell / 2, \ell / 2}$-cohomology only in positions 0,1 and 2 (see Theorem 4.20). On the other hand, we have the isomorphisms (see [Muk81])

$$
\Psi \circ \hat{\Psi} \cong(-1)^{*} \operatorname{id}_{D^{b}(X)}[-2] \quad \text { and } \quad \hat{\Psi} \circ \Psi \cong(-1)^{*} \operatorname{id}_{D^{b}(X)}[-2]
$$

Therefore the abelian category $\mathcal{B}_{\sqrt{3} \ell / 2, \ell / 2}$ behaves somewhat similarly to the category of coherent sheaves on an abelian surface under the Fourier-Mukai transform (see [BBR09, Mac96, Yos09] for further details). Now Theorem 4.20 becomes the key technical tool to show that the second tilt $\mathcal{A}_{\sqrt{3} \ell / 2, \ell / 2}$ is preserved by $\Psi$.

Under this auto-equivalence, minimal objects are mapped to minimal objects. This provides us with an inequality which bounds the top component of the Chern character of the object. This is the main idea for showing that the B-G-type inequality is satisfied by our restricted class of minimal objects in $\mathcal{A}_{\sqrt{3} \ell / 2, \ell / 2}$. In Section 5 , we have to show that the B-G-type inequality is satisfied by a very special class of minimal objects by showing that these objects actually do not exist. This result is of interest in its own right as it shows that if a bundle $E$ of such threefolds satisfies $c_{1}(E)=0=c_{2}(E)$, then it cannot carry a non-flat Hermitian-Einstein connection. 


\section{A. Maciocia and D. Piyaratne}

\section{Notation}

(i) For $0 \leqslant i \leqslant \operatorname{dim} X$, we set

$$
\begin{aligned}
\operatorname{Coh}^{\leqslant i}(X) & :=\{E \in \operatorname{Coh}(X): \operatorname{dim} \operatorname{Supp}(E) \leqslant i\}, \\
\operatorname{Coh}^{\geqslant i}(X) & :=\{E \in \operatorname{Coh}(X): \text { for } 0 \neq F \subset E, \operatorname{dim} \operatorname{Supp}(F) \geqslant i\}, \text { and } \\
\operatorname{Coh}^{i}(X) & :=\operatorname{Coh}^{\leqslant i}(X) \cap \operatorname{Coh}^{\geqslant i}(X) .
\end{aligned}
$$

(ii) For an interval $I \subset \mathbb{R} \cup\{+\infty\}$, we set $\operatorname{HN}_{\omega, B}^{\mu}(I):=\left\{E \in \operatorname{Coh}(X):\left[\mu_{\omega, B}^{-}(E), \mu_{\omega, B}^{+}(E)\right] \subset I\right\}$. We define the subcategory $\operatorname{HN}_{\omega, B}^{\nu}(I) \subset \mathcal{B}_{\omega, B}$ similarly.

(iii) For a Fourier-Mukai transform $\Upsilon$ and a heart $\mathfrak{A}$ of a t-structure for which $D^{b}(X) \cong D^{b}(\mathfrak{A})$, we set $\Upsilon_{\mathfrak{A}}^{k}(E):=H_{\mathfrak{A}}^{k}(\Upsilon(E))$.

(iv) For a sequence of integers $i_{1}, \ldots, i_{s}$, we set

$$
V_{\mathfrak{A}}^{\Upsilon}\left(i_{1}, \ldots, i_{s}\right):=\left\{E \in D^{b}(X): \Upsilon_{\mathfrak{A}}^{j}(E)=0 \text { for } j \notin\left\{i_{1}, \ldots, i_{s}\right\}\right\} .
$$

Then $E \in \operatorname{Coh}(X)$ being $\Upsilon$-WIT $i$ is equivalent to $E$ being an element of $V_{\operatorname{Coh}(X)}^{\Upsilon}(i)$.

(v) Let $(X, L)$ be a principally polarized abelian variety. Then we write $\Phi$ for the FMT from $X$ to $X$ with the Poincaré line bundle $\mathscr{P}:=m^{*} L \otimes p_{1}^{*} L^{-1} \otimes p_{2}^{*} L^{-1}$ on $X \times X$ as the kernel.

(vi) For $E \in \operatorname{Coh}(X)$, we set $E^{k}:=\Phi_{\operatorname{Coh}(X)}^{k}(E)$.

(vii) We set $\Psi:=L \Phi$ and $\hat{\Psi}:=\Phi L^{-1}[1]$. Here and elsewhere we abuse notation and write $L$ for the functor $L \otimes-$.

(viii) For a polarized projective threefold $(X, L)$ with Picard rank one over $\mathbb{C}$, the Chern character of $E$ is $\operatorname{ch}(E)=\left(a_{0}, a_{1} \ell, a_{2} \ell^{2} / 2, a_{3} \ell^{3} / 6\right)$ for some $a_{i} \in \mathbb{Q}$. For simplicity we write $\operatorname{ch}(E)=$ $\left(a_{0}, a_{1}, a_{2}, a_{3}\right)$. The $a_{i}$ are integers for the principally polarized abelian threefold case.

\section{Preliminaries}

\subsection{Construction of stability conditions}

We recall the conjectural construction of stability conditions as introduced in [BMT13].

Let $X$ be a smooth projective threefold over $\mathbb{C}$. Let $\omega, B$ be in $\mathrm{NS}_{\mathbb{R}}(X)$ with $\omega$ an ample class. The twisted Chern character $\operatorname{ch}^{B}$ with respect to $B$ is defined by $\operatorname{ch}^{B}(-)=e^{-B} \operatorname{ch}(-)$. So we have

$$
\begin{gathered}
\operatorname{ch}_{0}^{B}=\operatorname{ch}_{0}, \quad \operatorname{ch}_{1}^{B}=\operatorname{ch}_{1}-B \operatorname{ch}_{0}, \quad \operatorname{ch}_{2}^{B}=\operatorname{ch}_{2}-B \operatorname{ch}_{1}+\frac{1}{2} B^{2} \operatorname{ch}_{0} \\
\operatorname{ch}_{3}^{B}=\operatorname{ch}_{3}-B \operatorname{ch}_{2}+\frac{1}{2} B^{2} \operatorname{ch}_{1}-\frac{1}{6} B^{3} \operatorname{ch}_{0} .
\end{gathered}
$$

The twisted slope $\mu_{\omega, B}$ on $\operatorname{Coh}(X)$ is defined by

$$
\mu_{\omega, B}(E)= \begin{cases}+\infty & \text { if } E \text { is a torsion sheaf } \\ \omega^{2} \operatorname{ch}_{1}^{B}(E) / \operatorname{ch}_{0}^{B}(E) & \text { otherwise }\end{cases}
$$

for $E \in \operatorname{Coh}(X)$. Then $E$ is said to be $\mu_{\omega, B}$-stable (respectively, $\mu_{\omega, B}$-semistable), if for any $0 \neq F \varsubsetneqq E$, we have $\mu_{\omega, B}(F)<\mu_{\omega, B}(E / F)$ (respectively, $\mu_{\omega, B}(F) \leqslant \mu_{\omega, B}(E / F)$ ). The H-N filtration of $E$ with respect to $\mu_{\omega, B}$-stability enables us to define the following slopes:

$$
\mu_{\omega, B}^{+}(E)=\max _{0 \neq G \subseteq E} \mu_{\omega, B}(G), \quad \mu_{\omega, B}^{-}(E)=\min _{G \subsetneq E} \mu_{\omega, B}(E / G) .
$$




\section{FMTS AND STABILITY CONDITIONS ON ABELIAN THREEFOLDS}

For an interval $I \subset \mathbb{R} \cup\{+\infty\}$, the subcategory $\operatorname{HN}_{\omega, B}^{\mu}(I) \subset \operatorname{Coh}(X)$ is defined by

$$
\mathrm{HN}_{\omega, B}^{\mu}(I)=\left\{E \in \operatorname{Coh}(X):\left[\mu_{\omega, B}^{-}(E), \mu_{\omega, B}^{+}(E)\right] \subset I\right\} .
$$

Define the subcategories $\mathcal{T}_{\omega, B}$ and $\mathcal{F}_{\omega, B}$ of $\operatorname{Coh}(X)$ by setting

$$
\mathcal{T}_{\omega, B}=\operatorname{HN}_{\omega, B}^{\mu}(0,+\infty], \quad \mathcal{F}_{\omega, B}=\operatorname{HN}_{\omega, B}^{\mu}(-\infty, 0] .
$$

Then $\left(\mathcal{T}_{\omega, B}, \mathcal{F}_{\omega, B}\right)$ forms a torsion pair on $\operatorname{Coh}(X)$. The corresponding tilt of $\operatorname{Coh}(X)$ is the abelian category $\mathcal{B}_{\omega, B}=\left\langle\mathcal{F}_{\omega, B}[1], \mathcal{T}_{\omega, B}\right\rangle \subset D^{b}(X)$

The central charge function $Z_{\omega, B}: K(X) \rightarrow \mathbb{C}$ is defined by

$$
Z_{\omega, B}(E)=-\int_{X} e^{-B-i \omega} \operatorname{ch}(E) \text {. }
$$

So $\left.Z_{\omega, B}(E)=\left(-\operatorname{ch}_{3}^{B}(E)+\frac{1}{2} \omega^{2}\right) \operatorname{ch}_{1}^{B}(E)\right)+i\left(\omega \operatorname{ch}_{2}^{B}(E)-\frac{1}{6} \omega^{3} \operatorname{ch}_{0}^{B}(E)\right)$. The following result is very useful.

Lemma 1.1 ([BMT13, Lemma 3.2.1]). For any $0 \neq E \in \mathcal{B}_{\omega, B}$, one of the following conditions holds:

(i) $\omega^{2} \operatorname{ch}_{1}^{B}(E)>0$.

(ii) We have $\omega^{2} \operatorname{ch}_{1}^{B}(E)=0$ and $\Im\left(Z_{\omega, B}(E)\right)>0$.

(iii) We have $\omega^{2} \operatorname{ch}_{1}^{B}(E)=\Im\left(Z_{\omega, B}(E)\right)=0,-\Re\left(Z_{\omega, B}(E)\right)>0$ and $E \cong T$ for some $0 \neq T \in$ $\operatorname{Coh}^{0}(X)$.

As a result of this lemma, the authors of [BMT13] go on to remark that for objects in $\mathcal{B}_{\omega, B}$, the vector $\left(\omega^{2} \operatorname{ch}_{1}^{B}, \Im\left(Z_{\omega, B}\right)-\Re\left(Z_{\omega, B}\right)\right)$ behaves like the Chern character vector $\mathrm{ch}=\left(\mathrm{ch}_{0}, \mathrm{ch}_{1}, \mathrm{ch}_{2}\right)$ for coherent sheaves on a surface.

Following [BMT13], the tilt-slope $\nu_{\omega, B}$ on $\mathcal{B}_{\omega, B}$ is defined by

$$
\nu_{\omega, B}(E)= \begin{cases}+\infty & \text { if } \omega^{2} \operatorname{ch}_{1}^{B}(E)=0, \\ \Im\left(Z_{\omega, B}(E)\right) / \omega^{2} \operatorname{ch}_{1}^{B}(E) & \text { otherwise }\end{cases}
$$

for $E \in \mathcal{B}_{\omega, B}$. Then $E$ is said to be $\nu_{\omega, B}$-stable (respectively, $\nu_{\omega, B}$-semistable) if for any $0 \neq F \varsubsetneqq E$ in $\mathcal{B}_{\omega, B}$, we have $\nu_{\omega, B}(F)<\nu_{\omega, B}(E / F)$ (respectively, $\nu_{\omega, B}(F) \leqslant \nu_{\omega, B}(E / F)$ ). In [BMT13] it is proved that the abelian category $\mathcal{B}_{\omega, B}$ satisfies the $\mathrm{H}-\mathrm{N}$ property with respect to the tilt-slope stability. So the following slopes can be defined for $E \in \mathcal{B}_{\omega, B}$ :

$$
\nu_{\omega, B}^{+}(E)=\max _{0 \neq G \subseteq E} \nu_{\omega, B}(G), \quad \nu_{\omega, B}^{-}(E)=\min _{G \subsetneq E} \nu_{\omega, B}(E / G) .
$$

For an interval $I \subset \mathbb{R} \cup\{+\infty\}$, the subcategory $\operatorname{HN}_{\omega, B}^{\nu}(I) \subset \mathcal{B}_{\omega, B}$ is defined by

$$
\operatorname{HN}_{\omega, B}^{\nu}(I)=\left\{E \in \mathcal{B}_{\omega, B}:\left[\nu_{\omega, B}^{-}(E), \nu_{\omega, B}^{+}(E)\right] \subset I\right\}
$$

Define the subcategories $\mathcal{T}_{\omega, B}^{\prime}$ and $\mathcal{F}_{\omega, B}^{\prime}$ of $\mathcal{B}_{\omega, B}$ by setting

$$
\mathcal{T}_{\omega, B}^{\prime}=\mathrm{HN}_{\omega, B}^{\nu}(0,+\infty], \quad \mathcal{F}_{\omega, B}^{\prime}=\mathrm{HN}_{\omega, B}^{\nu}(-\infty, 0]
$$

Then $\left(\mathcal{T}_{\omega, B}^{\prime}, \mathcal{F}_{\omega, B}^{\prime}\right)$ forms a torsion pair on $\mathcal{B}_{\omega, B}$. The corresponding tilt of $\mathcal{B}_{\omega, B}$ is the abelian category $\mathcal{A}_{\omega, B}=\left\langle\mathcal{F}_{\omega, B}^{\prime}[1], \mathcal{T}_{\omega, B}^{\prime}\right\rangle \subset D^{b}(X)$.

Conjecture 1.2 ([BMT13, Conjecture 3.2.6]). The pair $\left(Z_{\omega, B}, \mathcal{A}_{\omega, B}\right)$ is a Bridgeland stability condition on $D^{b}(X)$. 


\section{A. Maciocia and D. Piyaratne}

Definition 1.3. Let $\mathscr{C}_{\omega, B}$ be the class of $\nu_{\omega, B}$-stable objects $E \in \mathcal{B}_{\omega, B}$ with $\nu_{\omega, B}(E)=0$.

We have $E[1] \in \mathcal{A}_{\omega, B}$ for any $E \in \mathscr{C}_{\omega, B}$.

Conjecture 1.4 ([BMT13, Conjecture 3.2.7]). Any $E \in \mathscr{C}_{\omega, B}$ satisfies the so-called BogomolovGieseker-type inequality:

$$
\Re\left(Z_{\omega, B}(E[1])\right)<0 \text {; that is, } \operatorname{ch}_{3}^{B}(E)<\frac{1}{2} \omega^{2} \operatorname{ch}_{1}^{B}(E) .
$$

Assume $B \in \mathrm{NS}_{\mathbb{Q}}(X)$ and let $\omega \in \mathrm{NS}_{\mathbb{R}}(X)$ be an ample class with $\omega^{2}$ rational. Then the abelian category $\mathcal{A}_{\omega, B}$ satisfies the important property stated in the following lemma. This was proved for rational classes $\omega$ in [BMT13]. However, a similar proof can be used when we have a weaker condition, namely $\omega^{2}$ rational. For example, a different parametrization given by $\omega \mapsto \sqrt{3} \omega$ is considered in [Mac12].

Lemma 1.5 ([BMT13, Proposition 5.2.2]). The abelian category $\mathcal{A}_{\omega, B}$ is Noetherian.

As a corollary we have the following result.

Corollary 1.6 ([BMT13, Corollary 5.2.4]). Conjectures 1.2 and 1.4 are equivalent.

\subsection{Fourier-Mukai transforms on abelian threefolds}

Let us quickly recall the notion of Fourier-Mukai transform on abelian threefolds. See [BBR09, Huy06] for further details on Fourier-Mukai theory.

Let $(X, L)$ be a principally polarized abelian threefold with Picard rank one. Let $\ell:=c_{1}(L)$. Then $\chi(L)=\ell^{3} / 6=1$. Let $\mathscr{P}=m^{*} L \otimes p_{1}^{*} L^{-1} \otimes p_{2}^{*} L^{-1}$ be the Poincaré line bundle on $X \times X$. Then the Fourier-Mukai transform $\Phi: D^{b}(X) \rightarrow D^{b}(X)$ with kernel $\mathscr{P}$ is defined by

$$
\Phi(-):=\mathbf{R} p_{2 *}\left(\mathscr{P} \stackrel{\mathbf{L}}{\otimes} p_{1}^{*}(-)\right) .
$$

Here $X \stackrel{p_{1}}{\longleftarrow} X \times X \stackrel{p_{2}}{\longrightarrow} X$ are the projection maps. In [Muk81] Mukai proved that $\Phi$ is an auto-equivalence of the derived category $D^{b}(X)$ and also that

$$
\Phi \circ \Phi \cong(-1)^{*} \operatorname{id}_{D^{b}(X)}[-3]
$$

The Chern character of any $E \in D^{b}(X)$ is of the form $\operatorname{ch}(E)=\left(a_{0}, a_{1} \ell, a_{2} \ell^{2} / 2, a_{3} \ell^{3} / 6\right)$ for some integers $a_{i}$. We have

(see [Huy06, Lemma 9.23]).

$$
\operatorname{ch}(\Phi(E))=\left(a_{3},-a_{2} \ell, a_{1} \ell^{2} / 2,-a_{0} \ell^{3} / 6\right)
$$

\section{Minimal objects of $\mathcal{A}_{\omega, B}$ and B-G-type inequality of threefolds}

\subsection{Some minimal objects of $\mathcal{A}_{\omega, B}$}

We identify some classes of minimal objects of the abelian category $\mathcal{A}_{\omega, B}$ of a projective threefold $X$. See [Huy08] for a detailed discussion on minimal objects of some abelian categories associated with Bridgeland stability conditions on a surface.

Proposition 2.1. For any $x \in X$, the skyscraper sheaf $\mathcal{O}_{x}$ is a minimal object in $\mathcal{A}_{\omega, B}$.

Proof. For any $x \in X$, we have $\mathcal{O}_{x} \in \mathcal{T}_{\omega, B}$ and also $\mathcal{O}_{x} \in \mathcal{T}_{\omega, B}^{\prime}$. Therefore $\mathcal{O}_{x} \in \mathcal{A}_{\omega, B}$. Let

$$
0 \rightarrow a \rightarrow \mathcal{O}_{x} \rightarrow b \rightarrow 0
$$




\section{FMTS AND STABILITY CONDITIONS ON ABELIAN THREEFOLDS}

be a short exact sequence in $\mathcal{A}_{\omega, B}$ such that $a \neq 0$. Then in order to prove that $\mathcal{O}_{x} \in \mathcal{A}_{\omega, B}$ is minimal, we need to show $b=0$. We obtain the following long exact sequence of $\mathcal{B}_{\omega, B}$-cohomology associated with the $\mathcal{A}_{\omega, B}$-short exact sequence above:

$$
0 \rightarrow A_{-1} \rightarrow 0 \rightarrow B_{-1} \rightarrow A_{0} \rightarrow \mathcal{O}_{x} \rightarrow B_{0} \rightarrow 0 \text {. }
$$

Here $A_{k}:=H_{\mathcal{B}_{\omega, B}}^{k}(a)$ and $B_{k}:=H_{\mathcal{B}_{\omega, B}}^{k}(b)$. We have $A_{-1}=0$ and so $a \cong A_{0} \neq 0$. Let $C:=$ $A_{0} / B_{-1}$. Then

$$
0 \rightarrow C \rightarrow \mathcal{O}_{x} \rightarrow B_{0} \rightarrow 0
$$

is a short exact sequence in $\mathcal{B}_{\omega, B}$. We obtain the following long exact sequence of $\operatorname{Coh}(X)$ cohomology associated with the $\mathcal{B}_{\omega, B}$-short exact sequence above:

$$
0 \rightarrow C^{-1} \rightarrow 0 \rightarrow B_{0}^{-1} \rightarrow C^{0} \rightarrow \mathcal{O}_{x} \rightarrow B_{0}^{0} \rightarrow 0 .
$$

Here $C^{k}:=H_{\mathrm{Coh}(X)}^{k}(C)$ and $B_{0}^{k}:=H_{\mathrm{Coh}(X)}^{k}\left(B_{0}\right)$. We have $C^{-1}=0$ and so $C \cong C^{0}$.

If $B_{0}^{0} \neq 0$, then $\mathcal{O}_{x} \cong B_{0}^{0}$ and $B_{0}^{-1} \cong C^{0} \in \mathcal{T}_{\omega, B} \cap \mathcal{F}_{\omega, B}=\{0\}$. So $C=0$ and $B_{-1} \cong$ $A_{0} \in \mathcal{T}_{\omega, B}^{\prime} \cap \mathcal{F}_{\omega, B}^{\prime}=\{0\}$, which implies $A_{0}=0$. This is not possible and so $B_{0}^{0}=0$. Therefore $B_{0} \cong B_{0}^{-1}[1]$ and

$$
0 \rightarrow B_{0}^{-1} \rightarrow C^{0} \rightarrow \mathcal{O}_{x} \rightarrow 0
$$

is a short exact sequence in $\operatorname{Coh}(X)$. Here $\operatorname{ch}\left(\mathcal{O}_{x}\right)=(0,0,0,1)$. If $B_{0}^{-1} \neq 0$, then

$$
0 \geqslant \mu_{\omega, B}\left(B_{0}^{-1}\right)=\mu_{\omega, B}\left(C^{0}\right)>0 .
$$

This is not possible and so $B_{0}^{-1}=0$ and $C^{0} \cong \mathcal{O}_{x}$. Therefore $b \cong B_{-1}[1]$, and we have the following short exact sequence in $\mathcal{B}_{\omega, B}$ :

$$
0 \rightarrow B_{-1} \rightarrow A_{0} \rightarrow \mathcal{O}_{x} \rightarrow 0 \text {. }
$$

Since $\operatorname{ch}\left(\mathcal{O}_{x}\right)=(0,0,0,1)$, if $B_{-1} \neq 0$, then

$$
0 \geqslant \nu_{\omega, B}\left(B_{-1}\right)=\nu_{\omega, B}\left(A_{0}\right)>0 .
$$

This is not possible and so $B_{-1}=0$. Therefore $b=0$ and so $\mathcal{O}_{x} \in \mathcal{A}_{\omega, B}$ is a minimal object, as required.

We now identify further minimal objects.

Definition 2.2. Let $\mathscr{M}_{\omega, B}$ be the class of all objects $E \in \mathcal{B}_{\omega, B}$ such that

(i) the object $E$ is $\nu_{\omega, B}$-stable;

(ii) we have $\nu_{\omega, B}(E)=0$; and

(iii) we have $\operatorname{Ext}^{1}\left(\mathcal{O}_{x}, E\right)=0$ for any skyscraper sheaf $\mathcal{O}_{x}$ with $x \in X$.

We clearly have $\mathscr{M}_{\omega, B} \subset \mathscr{C}_{\omega, B}$.

Lemma 2.3. Let $E \in \mathscr{M}_{\omega, B}$. Then $E[1]$ is a minimal object of $\mathcal{A}_{\omega, B}$.

Proof. By definition $\mathscr{M}_{\omega, B} \subset \mathcal{F}_{\omega, B}^{\prime}$ and so $E[1] \in \mathcal{A}_{\omega, B}$. Let

$$
0 \rightarrow a \rightarrow E[1] \rightarrow b \rightarrow 0
$$

be a short exact sequence in $\mathcal{A}_{\omega, B}$ such that $b \neq 0$. Now we need to show $a=0$ or, equivalently, $b \cong E[1]$. We have the following long exact sequence of $\mathcal{B}_{\omega, B}$-cohomology associated with the $\mathcal{A}_{\omega, B}$-short exact sequence above:

$$
0 \rightarrow A_{-1} \rightarrow E \rightarrow B_{-1} \rightarrow A_{0} \rightarrow 0 \rightarrow B_{0} \rightarrow 0 .
$$




\section{A. Maciocia and D. Piyaratne}

Here $A_{k}:=H_{\mathcal{B}_{\omega, B}}^{k}(a)$ and $B_{k}:=H_{\mathcal{B}_{\omega, B}}^{k}(b)$. We have $B_{0}=0$ and so $b \cong B_{-1}[1]$, which implies $B_{-1} \neq 0$.

(i) Suppose $A_{-1} \neq 0$. If $E / A_{-1} \neq 0$, then $E / A_{-1} \hookrightarrow B_{-1}$ and $\nu_{\omega, B}^{+}\left(B_{-1}\right) \leqslant 0$ implies $\nu_{\omega, B}\left(E / A_{-1}\right) \leqslant 0$. On the other hand, $\nu_{\omega, B}\left(E / A_{-1}\right)>0$ as $A_{-1} \neq 0$ and $E$ is $\nu_{\omega, B}$-stable with $\nu_{\omega, B}(E)=0$. But this not possible.

If $E / A_{-1}=0$, then $A_{-1} \cong E$ and $B_{-1} \cong A_{0} \in \mathcal{F}_{\omega, B}^{\prime} \cap \mathcal{T}_{\omega, B}^{\prime}=\{0\}$. This is not possible as $B_{-1} \neq 0$.

(ii) Suppose $A_{-1}=0$. Then we have the following short exact sequence in $\mathcal{B}_{\omega, B}$ :

$$
0 \rightarrow E \rightarrow B_{-1} \rightarrow A_{0} \rightarrow 0 .
$$

If $A_{0} \neq 0$, then $\nu_{\omega, B}(E)=0$ implies $\omega^{2} \operatorname{ch}_{1}^{B}(E)>0$ and $\Im\left(Z_{\omega, B}(E)\right)=0$. Then

$$
\nu_{\omega, B}\left(B_{-1}\right)=\frac{\Im\left(Z_{\omega, B}\left(A_{0}\right)\right)}{\omega^{2} \operatorname{ch}_{1}^{B}(E)+\omega^{2} \operatorname{ch}_{1}^{B}\left(A_{0}\right)} \leqslant 0
$$

implies $\Im\left(Z_{\omega, B}\left(A_{0}\right)\right) \leqslant 0$. If $\omega^{2} \operatorname{ch}_{1}^{B}\left(A_{0}\right) \neq 0$, then $\nu_{\omega, B}\left(A_{0}\right)>0$ implies $\Im\left(Z_{\omega, B}\left(A_{0}\right)\right)>0$, which is not possible. Hence $\omega^{2} \operatorname{ch}_{1}^{B}\left(A_{0}\right)=0$ and by Lemma 1.1, $\Im\left(Z_{\omega, B}\left(A_{0}\right)\right) \geqslant 0$. So $\Im\left(Z_{\omega, B}\left(A_{0}\right)\right)=0$ and $A_{0} \cong T$ for some $0 \neq T \in \operatorname{Coh}^{0}(X)$. Then the $\mathcal{B}_{\omega, B^{-}}$-short exact sequence (2.1) corresponds to an element from $\operatorname{Ext}^{1}\left(A_{0}, E\right)=\operatorname{Ext}^{1}(T, E)$. But we have $\operatorname{Ext}^{1}\left(\mathcal{O}_{x}, E\right)=0$ for any $x \in X$ and so $\operatorname{Ext}^{1}(T, E)=0$. Hence $B_{-1} \cong T \oplus E$. Then $T$ is a subobject of $B_{-1}$. But this is not possible as $\nu_{\omega, B}(T)=+\infty$ and $E \in \mathscr{M}_{\omega, B}$.

If $A_{0}=0$, then $a=0$ and $b \cong B_{-1}[1] \cong E[1]$, as required.

This completes the proof of the lemma.

Some classes of tilt-stable candidates have been identified in [BMT13].

Recall that for $E \in D^{b}(X)$, the discriminant $\bar{\Delta}_{\omega}$ in the sense of Drézet is defined by

$$
\bar{\Delta}_{\omega}(E)=\left(\omega^{2} \operatorname{ch}_{1}^{B}(E)\right)^{2}-2 \omega^{3} \operatorname{ch}_{0}^{B}(E) \cdot \omega \operatorname{ch}_{2}^{B}(E) .
$$

Proposition 2.4 ([BMT13, Proposition 7.4.1]). Let $E$ be a $\mu_{\omega, B}$-stable locally free sheaf on $X$ with $\bar{\Delta}_{\omega}(E)=0$. Then either $E$ or $E[1]$ in $\mathcal{B}_{\omega, B}$ is $\nu_{\omega, B}$-stable.

Example 2.5. Let $(X, L)$ be a polarized projective threefold and let $\ell:=c_{1}(L)$. Consider the classes $B=\ell / 2$ and $\omega=\sqrt{3} \ell / 2$. Then $\bar{\Delta}_{\omega}(\mathcal{O})=\bar{\Delta}_{\omega}(L)=0$. So, by Proposition 2.4 , the elements $\mathcal{O}[1]$ and $L$ of $\mathcal{B}_{\omega, B}$ are $\nu_{\omega, B}$-stable. We also have $\Im\left(Z_{\omega, B}(\mathcal{O}[1])\right)=\Im\left(Z_{\omega, B}(L)\right)=0$. Therefore $\nu_{\omega, B}(\mathcal{O}[1])=\nu_{\omega, B}(L)=0$. So by Lemma 2.3 , the objects $\mathcal{O}[2]$ and $L[1]$ in $\mathcal{A}_{\omega, B}$ are minimal.

Remark 2.6. The tilt stable objects associated with minimal objects in Example 2.5 clearly satisfy the corresponding B-G-type inequalities.

\subsection{Reduction of B-G-type inequalities for minimal objects}

The following propositions play an important role in this paper.

Proposition 2.7 ([LM12, Proposition 3.1]). Let $E \in \mathcal{B}_{\omega, B}$ be a $\nu_{\omega, B}$-semistable object with $\nu_{\omega, B}<+\infty$. Then $H_{\mathrm{Coh}(X)}^{-1}(E)$ is a reflexive sheaf.

Proposition 2.8 ([LM12, Proposition 3.5]). Let $0 \rightarrow E \rightarrow E^{\prime} \rightarrow Q \rightarrow 0$ be a non-splitting short exact sequence in $\mathcal{B}_{\omega, B}$ with $Q \in \operatorname{Coh}^{0}(X), \operatorname{Hom}\left(\mathcal{O}_{x}, E^{\prime}\right)=0$ for all $x \in X$ and $\omega^{2} \operatorname{ch}_{1}^{B}(E) \neq 0$. If $E$ is $\nu_{\omega, B}$-stable, then $E^{\prime}$ is $\nu_{\omega, B}$-stable. 


\section{FMTS AND STABILITY CONDITIONS ON ABELIAN THREEFOLDS}

Recall that $\mathscr{C}_{\omega, B}$ is the class of $\nu_{\omega, B}$-stable objects $E \in \mathcal{B}_{\omega, B}$ with $\nu_{\omega, B}(E)=0$.

Proposition 2.9. Let $E \in \mathscr{C}_{\omega, B}$. Then there exists an $E^{\prime} \in \mathscr{M}_{\omega, B}$ (that is, $E^{\prime}[1]$ is a minimal object of $\mathcal{A}_{\omega, B}$ ) such that

$$
0 \rightarrow E \rightarrow E^{\prime} \rightarrow Q \rightarrow 0
$$

is a short exact sequence in $\mathcal{B}_{\omega, B}$ for some $Q \in \operatorname{Coh}^{0}(X)$.

Proof. Let $E \in \mathscr{C}_{\omega, B} \backslash \mathscr{M}_{\omega, B}$. Assume the opposite of the claim in the proposition for $E$. Then there exists a sequence of non-splitting short exact sequences in $\mathcal{B}_{\omega, B}$ for $i \geqslant 1$,

$$
0 \rightarrow E_{i-1} \rightarrow E_{i} \rightarrow \mathcal{O}_{y_{i}} \rightarrow 0,
$$

where $E_{0}=E, E_{i} \in \mathscr{C}_{\omega, B}$ (see Proposition 2.8). So for each $i \geqslant 1$,

$$
0 \rightarrow \mathcal{O}_{y_{i}} \rightarrow E_{i-1}[1] \rightarrow E_{i}[1] \rightarrow 0
$$

is a short exact sequence in $\mathcal{A}_{\omega, B}$. Therefore

$$
E[1]=E_{0}[1] \rightarrow E_{1}[1] \rightarrow E_{2}[1] \rightarrow \cdots
$$

is an infinite chain of quotients in $\mathcal{A}_{\omega, B}$. But this is not possible as $\mathcal{A}_{\omega, B}$ is Noetherian by Lemma 1.5. This is a contradiction.

It follows that $E \in \mathscr{C}_{\omega, B}$ satisfies the B-G-type inequality if the corresponding $E^{\prime} \in \mathscr{M}_{\omega, B}$ satisfies the B-G-type inequality.

\section{The abelian category $\mathcal{A}_{\sqrt{3} B, B}$, Fourier-Mukai transforms and stability conditions}

\subsection{Some properties of $\mathcal{A}_{\sqrt{3} B, B}$}

We discuss some of the properties of the abelian category $\mathcal{A}_{\sqrt{3} B, B}$ for an arbitrary polarized projective threefold $(X, L)$ with Picard rank one. Let $\ell:=c_{1}(L)$. Let $B=b \ell$ for $b \in \mathbb{Q}_{>0}$. Then for $E \in D^{b}(X)$, we have

$$
\Im\left(Z_{\sqrt{3} B, B}(E)\right)=\sqrt{3} b \ell\left(\operatorname{ch}_{2}(E)-b \ell \operatorname{ch}_{1}(E)\right) .
$$

Proposition 3.1. Let $E \in \mathcal{B}_{\sqrt{3} B, B}$ and let $E_{i}=H_{\operatorname{Coh}(X)}^{i}(E)$. Let $E_{i}^{ \pm}$be the $H$-N-semistable factors of $E_{i}$ with highest and lowest $\mu_{\sqrt{3} B, B}$-slopes. Then we have the following results:

(i) If $E \in \mathrm{HN}_{\sqrt{3} B, B}^{\nu}(-\infty, 0)$ and $E_{-1} \neq 0$, then $\ell^{2} \operatorname{ch}_{1}\left(E_{-1}^{+}\right)<0$.

(ii) If $E \in \mathrm{HN}_{\sqrt{3} B, B}^{\nu}(0,+\infty]$ and $\operatorname{rk}\left(E_{0}\right) \neq 0$, then $\ell^{2} \operatorname{ch}_{1}\left(E_{0}^{-}\right)>2 b \ell^{3} \operatorname{ch}_{0}\left(E_{0}^{-}\right)$.

(iii) If $E$ is tilt-semistable with $\nu_{\sqrt{3} B, B}(E)=0$, then

(a) for $E_{-1} \neq 0$, we have $\ell^{2} \operatorname{ch}_{1}\left(E_{-1}\right) \leqslant 0$ with equality if and only if $\operatorname{ch}_{2}\left(E_{-1}\right)=0$;

(b) for $\operatorname{rk}\left(E_{0}\right) \neq 0$, we have $\ell^{2} \operatorname{ch}_{1}\left(E_{0}\right) \geqslant 2 b \ell^{3} \operatorname{ch}_{0}\left(E_{0}\right)$, with equality if and only if $\left(\operatorname{ch}_{1}\left(E_{0}\right)\right)^{2}=2 \operatorname{ch}_{0}\left(E_{0}\right) \operatorname{ch}_{2}\left(E_{0}\right)$.

Proof. The object $E \in \mathcal{B}_{\sqrt{3} B, B}$ fits into the $\mathcal{B}_{\sqrt{3} B, B}$-short exact sequence

$$
0 \rightarrow E_{-1}[1] \rightarrow E \rightarrow E_{0} \rightarrow 0 .
$$

(i) Since $E$ is an element of $\operatorname{HN}_{\sqrt{3} B, B}^{\nu}(-\infty, 0)$, we have $E_{-1}[1] \in \operatorname{HN}_{\sqrt{3} B, B}^{\nu}(-\infty, 0)$. We also have $0 \neq E_{-1}^{+} \subseteq E_{-1}$. Hence $E_{-1}^{+}[1] \in \operatorname{HN}_{\sqrt{3} B, B}^{\nu}(-\infty, 0)$. 


\section{A. Maciocia and D. Piyaratne}

Let $\operatorname{ch}\left(E_{-1}^{+}\right)=\left(a_{0}, a_{1}, a_{2}, a_{3}\right)$. Assume the opposite of statement (i) for a contradiction, so that $a_{1} \geqslant 0$. We have

$$
\begin{aligned}
\nu_{\sqrt{3} B, B}\left(E_{-1}^{+}[1]\right) & =\frac{-\Im\left(Z_{\sqrt{3} B, B}\left(E_{-1}^{+}\right)\right)}{-3 B^{2} \operatorname{ch}_{1}^{B}\left(E_{-1}^{+}\right)} \\
& =\frac{\sqrt{3} b a_{1}\left(b a_{0}-a_{1}\right)+\frac{1}{2} \sqrt{3} b a_{1}^{2}+\frac{1}{2} \sqrt{3} b\left(a_{1}^{2}-a_{0} a_{2}\right)}{3 a_{0} b^{2}\left(b a_{0}-a_{1}\right)} .
\end{aligned}
$$

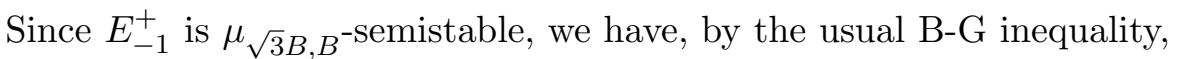

$$
a_{1}^{2}-a_{0} a_{2} \geqslant 0
$$

and since $E_{-1}^{+} \in \mathcal{F}_{\sqrt{3} B, B}$, we have $\nu_{\sqrt{3} B, B}\left(E_{-1}^{+}[1]\right) \neq+\infty$ and so $b a_{0}-a_{1}>0$. Hence, as $a_{0}>0$, we have $\nu_{\sqrt{3} B, B}\left(E_{-1}^{+}[1]\right) \geqslant 0$. But this is not possible as $E_{-1}^{+}[1] \in \operatorname{HN}_{\sqrt{3} B, B}^{\nu}(-\infty, 0)$. This is the contradiction required to complete the proof.

(ii) Since $E$ is an element of $\operatorname{HN}_{\sqrt{3} B, B}^{\nu}(0,+\infty]$, we have $E_{0} \in \operatorname{HN}_{\sqrt{3} B, B}^{\nu}(0,+\infty]$. The element $0 \neq E_{0}^{-}$is a torsion-free quotient of $E_{0}$. Since $E_{0}$ is an element of $\operatorname{HN}_{\sqrt{3} B, B}^{\nu}(0,+\infty]$, we have $E_{0}^{-} \in \mathrm{HN}_{\sqrt{3} B, B}^{\nu}(0,+\infty]$.

Let $\operatorname{ch}\left(E_{0}^{-}\right)=\left(a_{0}, a_{1}, a_{2}, a_{3}\right)$. Assume the opposite of statement (ii) for a contradiction, so that $a_{1} \leqslant 2 b a_{0}$. We have

$$
\begin{aligned}
\nu_{\sqrt{3} B, B}\left(E_{0}^{-}\right) & =\frac{\Im\left(Z_{\sqrt{3} B, B}\left(E_{0}^{-}\right)\right)}{3 B^{2} \operatorname{ch}_{1}^{B}\left(E_{0}^{-}\right)} \\
& =\frac{-\frac{1}{2} \sqrt{3} b\left(a_{1}^{2}-a_{0} a_{2}\right)+\frac{1}{2} \sqrt{3} b a_{1}\left(a_{1}-2 b a_{0}\right)}{3 b^{2} a_{0}\left(a_{1}-b a_{0}\right)} .
\end{aligned}
$$

Here $E_{0}^{-} \in \mathcal{T}_{\sqrt{3} B, B}$ is torsion free, which implies

$$
a_{1}-b a_{0}>0 .
$$

The object $E_{0}^{-}$is $\mu_{\sqrt{3} B, B^{-s e m i s t a b l e, ~ w h i c h ~ i m p l i e s ~(b y ~ t h e ~ u s u a l ~ B-G ~ i n e q u a l i t y) ~}}$

$$
a_{1}^{2}-a_{0} a_{2} \geqslant 0 \text {. }
$$

Therefore $\nu_{\sqrt{3} B, B}\left(E_{0}^{-}\right) \leqslant 0$. But this is not possible as $E_{0}^{-} \in \operatorname{HN}_{\sqrt{3} B, B}^{\nu}(0,+\infty]$. This is the contradiction required to complete the proof.

(iii) As in the proof of statement (i), one can show that if $E \in \mathrm{HN}_{\sqrt{3} B, B}^{\nu}(-\infty, 0]$ and $E_{-1} \neq 0$, then $\ell^{2} \operatorname{ch}_{1}\left(E_{-1}^{+}\right) \leqslant 0$. Hence, for $E \in \mathrm{HN}_{\sqrt{3} B, B}^{\nu}[0]$, we have $\ell^{2} \operatorname{ch}_{1}\left(E_{-1}\right) \leqslant 0$. The equality holds if and only if $E_{-1}$ is slope-semistable, and so it satisfies the usual B-G inequality. Since $\nu_{\sqrt{3} B, B}\left(E_{-1}\right) \leqslant 0$, we have $\ell^{2} \operatorname{ch}_{1}\left(E_{-1}\right)=0$ if and only if $\operatorname{ch}_{2}\left(E_{-1}\right)=0$.

The proof of assertion (b) is similar to that of assertion (a).

\subsection{The relation of Fourier-Mukai transforms to stability conditions}

Let $(X, L)$ be a principally polarized abelian threefold with Picard rank one. Let $\ell:=c_{1}(L)$. Then $\chi(L)=\ell^{3} / 6=1$ and the Chern character of $E \in D^{b}(X)$ is of the form $\operatorname{ch}(E)=$ $\left(a_{0}, a_{1} \ell, a_{2} \ell^{2} / 2, a_{3} \ell^{3} / 6\right)$ for some integers $a_{i}$. Define the classes $B=\ell / 2$ and $\omega=\sqrt{3} \ell / 2$.

The following is a key result in this paper.

Proposition 3.2. If $\Phi\left(L^{-1} E\right)[2] \in \mathcal{B}_{\omega, B}$ for any $E \in \mathscr{M}_{\omega, B} \backslash\left\{L \mathscr{P}_{x}: x \in X\right\}$, then the B-G-type inequality holds for the objects in $\mathscr{C}_{\omega, B}$. 


\section{FMTS AND STABILITY CONDITIONS ON ABELIAN THREEFOLDS}

Proof. By Proposition 2.9, it is enough to check that the B-G-type inequality is satisfied by each object in $\mathscr{M}_{\omega, B}$. Moreover, the objects in $\left\{L \mathscr{P}_{x}: x \in X\right\} \subset \mathscr{M}_{\omega, B}$ satisfy the B-G-type inequality (see Remark 2.6). Hence we only need to check the inequality for objects in the set $\mathscr{M}_{\omega, B} \backslash\left\{L \mathscr{P}_{x}: x \in X\right\}$.

Let $E \in \mathscr{M}_{\omega, B} \backslash\left\{L \mathscr{P}_{x}: x \in X\right\}$ and assume that $\Phi\left(L^{-1} E\right)[2]$ is an element of $\mathcal{B}_{\omega, B}$. Let $\operatorname{ch}(E)=\left(a_{0}, a_{1} \ell, a_{2} \ell^{2} / 2, a_{3} \ell^{3} / 6\right)$. Then $\Im\left(Z_{\omega, B}(E)\right)=0$ implies $a_{1}=a_{2}$. Now the B-G-type inequality says

$$
\Delta:=-a_{0}+3 a_{1}-a_{3}>0 .
$$

By Proposition 3.1, we have $\ell^{2} \operatorname{ch}_{1}\left(E_{-1}\right) \leqslant 0$ and $\ell^{2} \operatorname{ch}_{1}\left(E_{0}\right) \geqslant 0$, where $E_{i}=H_{\operatorname{Coh}(X)}^{i}(E)$. So $a_{1} \ell^{3}=\ell^{2} \operatorname{ch}_{1}(E)=\ell^{2} \operatorname{ch}_{1}\left(E_{0}\right)-\ell^{2} \operatorname{ch}_{1}\left(E_{-1}\right) \geqslant 0$.

Let $F=\Phi\left(L^{-1} E\right)[2]$ and let $\operatorname{ch}(F)=\left(b_{0}, b_{1} \ell, b_{2} \ell^{2} / 2, b_{3} \ell^{3} / 6\right)$. Then $b_{0}=a_{3}-a_{0}$ and $b_{1}=$ $b_{2}=a_{1}-a_{0}$. Now $b_{1}=b_{2}$ implies $\Im\left(Z_{\omega, B}(F)\right)=0$. Also, $F \in \mathcal{B}_{\omega, B}$ implies $\omega^{2} \operatorname{ch}_{1}^{B}(F) \geqslant 0$; that is, $2 b_{1}-b_{0} \geqslant 0$. If $\omega^{2} \operatorname{ch}_{1}^{B}(F)=0$, then $\Im\left(Z_{\omega, B}(F)\right)=0$ implies $F \cong T$ for some $T \in \operatorname{Coh}^{0}(X)$ (see Lemma 1.1). If $T \neq 0$, then $E$ has a filtration with factors of the form $L \mathscr{P}_{x}[1] \notin \mathscr{M}_{\omega, B}$. This is not possible and so $\omega^{2} \operatorname{ch}_{1}^{B}(F)>0$; that is, $2 b_{1}-b_{0}=-a_{0}+2 a_{1}-a_{3}>0$. Hence $\Delta>0$ and so $E$ satisfies the B-G-type inequality. This completes the proof.

Our main goal in the rest of this paper is to prove that $\Phi L^{-1}[2]$ and its quasi-inverse $L \Phi[1]$ are auto-equivalences of the abelian category $\mathcal{A}_{\omega, B}$. Under an equivalence of abelian categories minimal objects are mapped to minimal objects and so the hypothesis of Proposition 3.2 is satisfied. Therefore, by Corollary 1.6, we have the following result.

Theorem 3.3. The pair $\left(\mathcal{A}_{\omega, B}, Z_{\omega, B}\right)$ is a Bridgeland stability condition on $D^{b}(X)$.

\section{Fourier-Mukai transforms on the sheaves $\operatorname{Coh}(X)$ of abelian threefolds}

From here onward, we always assume that $(X, L)$ is a principally polarized abelian threefold with Picard rank one. Let $\ell:=c_{1}(L)$. Then $\chi(L)=\ell^{3} / 6=1$ and the Chern character of any $E \in D^{b}(X)$ is of the form $\operatorname{ch}(E)=\left(a_{0}, a_{1} \ell, a_{2} \ell^{2} / 2, a_{3} \ell^{3} / 2\right)$ for some integers $a_{i}$. Define the classes $B=\ell / 2$ and $\omega=\sqrt{3} \ell / 2$.

If $E \in \operatorname{Coh}(X)$, then the slope $\mu(E)$ is defined by $\mu(E):=\mu_{\ell / \sqrt{6}, 0}(E)$; that is, $\mu(E)=a_{1} / a_{0}$ when $a_{0} \neq 0$ and $\mu(E)=+\infty$ when $a_{0}=0$. In the rest of the paper we mostly use $\mu$-slope for coherent sheaves and we simply write $\mathrm{HN}$ for $\mathrm{HN}_{\ell / \sqrt{6}, 0}^{\mu}$. Then $\mu_{\omega, B}(E)=\frac{9}{2}\left(\mu(E)-\frac{1}{2}\right)$. Moreover, define $\mathcal{T}_{0}=\mathrm{HN}(0,+\infty]$ and $\mathcal{F}_{0}=\operatorname{HN}(-\infty, 0]$. For simplicity, we write $\mathcal{T}=\mathcal{T}_{\omega, B}, \mathcal{F}=\mathcal{F}_{\omega, B}$, $\mathcal{B}=\mathcal{B}_{\omega, B}, \nu=\nu_{\omega, B}, \mathrm{HN}_{\omega, B}^{\nu}=\mathrm{HN}^{\nu}, \mathcal{T}^{\prime}=\mathcal{T}_{\omega, B}^{\prime}, \mathcal{F}^{\prime}=\mathcal{F}_{\omega, B}^{\prime}$ and $\mathcal{A}=\mathcal{A}_{\omega, B}$. By the definitions, we have $\mathcal{F}=\operatorname{HN}\left(-\infty, \frac{1}{2}\right]$ and $\mathcal{T}=\operatorname{HN}\left(\frac{1}{2},+\infty\right]$.

Let $\Phi$ be the FMT with kernel the Poincaré line bundle $\mathscr{P}$. The isomorphism $\Phi \circ \Phi \cong$ $(-1)^{*} \operatorname{id}_{\operatorname{id}_{D^{b}(X)}}[-3]$ gives us the following convergence of a spectral sequence.

Proposition 4.1 (Mukai spectral sequence). We have

$$
E_{2}^{p, q}=\Phi_{\operatorname{Coh}(X)}^{p} \Phi_{\operatorname{Coh}(X)}^{q}(E) \Longrightarrow H_{\operatorname{Coh}(X)}^{p+q-3}\left((-1)^{*} E\right)
$$

for $E \in \operatorname{Coh}(X)$, where $\Phi_{\operatorname{Coh}(X)}^{i}(F)=H_{\operatorname{Coh}(X)}^{i}(\Phi(F))$.

For $E \in \operatorname{Coh}(X)$, we write

$$
E^{k}=\Phi_{\mathrm{Coh}(X)}^{k}(E)
$$




\section{A. Maciocia and D. Piyaratne}

Then for example $E^{120}=\Phi_{\operatorname{Coh}(X)}^{0} \Phi_{\operatorname{Coh}(X)}^{2} \Phi_{\operatorname{Coh}(X)}^{1}(E)$. Using this notation, we can deduce the following immediately from the spectral sequence:

$$
E^{00}=E^{01}=E^{32}=E^{33}=0, \quad E^{10} \cong E^{02} \quad \text { and } \quad E^{31} \cong E^{23} .
$$

Let $\mathbf{R} \Delta$ denote the derived dualizing functor $\mathbf{R} \mathcal{H}$ om $(-, \mathcal{O})[3]$. Then by $[\operatorname{Muk} 81,(3.8)]$, we have

$$
(\Phi \circ \mathbf{R} \Delta)[3] \cong(-1)^{*} \mathbf{R} \Delta \circ \Phi .
$$

This gives us the convergence of the following spectral sequences.

Proposition 4.2 (Duality spectral sequence). For any $E \in \operatorname{Coh}(X)$ we have two spectral sequences

$$
\Phi_{\operatorname{Coh}(X)}^{p}\left(\mathcal{E} x t^{q+3}(E, \mathcal{O})\right) \quad \text { and } \quad(-1)^{*} \mathcal{E} x t^{p+3}\left(\Phi_{\operatorname{Coh}(X)}^{3-q}(E), \mathcal{O}\right)
$$

which are co-convergent.

The aim of this section is to use mainly the Mukai and duality spectral sequences to study the slope stability of sheaves under the Fourier-Mukai transform $\Phi$. More precisely, we consider the $\operatorname{Coh}(X)$-cohomology sheaves of the images under $\Phi$ of torsion sheaves supported in dimensions one and two. We also study the transforms of torsion-free sheaves whose H-N-semistable factors satisfy certain slope bounds.

Notation 4.3. Any $E \in \operatorname{Coh}(X)$ fits into $\operatorname{Coh}(X)$-short exact sequence

$$
0 \rightarrow T \rightarrow E \rightarrow F \rightarrow 0
$$

for some $T \in \mathcal{T}_{0}$ and $F \in \mathcal{F}_{0}$. Denote $T(E)$ by $T$ and $F(E)$ by $F$.

Any torsion-free sheaf $E$ fits into a non-splitting $\operatorname{Coh}(X)$-short exact sequence

$$
0 \rightarrow E \rightarrow E^{* *} \rightarrow T \rightarrow 0
$$

for some $T \in \mathrm{Coh}^{\leqslant 1}(X)$. Here $E^{* *}$ is a reflexive sheaf. If $E$ has rank one, then $E^{* *}$ is a line bundle and so $E^{* *} \cong L^{k} \mathscr{P}_{x}$ for some $k \in \mathbb{Z}$ and $x \in X$.

Notation 4.4. If $E$ is a rank one torsion-free sheaf with $c_{1}(E)=k \ell$, then we can write $E=$ $L^{k} \mathscr{P}_{x} \mathcal{I}_{C}$. Here $\mathcal{I}_{C}$ is the ideal sheaf of the structure sheaf $\mathcal{O}_{C}:=L^{-k} \mathscr{P}_{-x} \otimes\left(E^{* *} / E\right) \in \mathrm{Coh}^{\leqslant 1}(X)$ of a subscheme $C \subset X$ of dimension at most one.

Proposition 4.5. Let $E \in \operatorname{Coh}(X)$. If $E^{0} \neq 0$, then $E^{0}$ is a reflexive sheaf.

Proof. Let $x \in X$. Then for $0 \leqslant i \leqslant 2$, we have

$$
\operatorname{Hom}\left(\mathcal{O}_{x}, E^{0}[i]\right) \cong \operatorname{Hom}\left(\Phi\left(\mathcal{O}_{x}\right), \Phi\left(E^{0}\right)[i]\right) \cong \operatorname{Hom}\left(\mathscr{P}_{x}, E^{02}[-2+i]\right)
$$

from the convergence of the Mukai spectral sequence (4.1) for $E$. Consequently, $\operatorname{Hom}\left(\mathcal{O}_{x}, E^{0}\right)=$ $\operatorname{Ext}^{1}\left(\mathcal{O}_{x}, E^{0}\right)=0$, and

$$
\begin{aligned}
\operatorname{Ext}^{2}\left(\mathcal{O}_{x}, E^{0}\right) & \cong \operatorname{Hom}\left(\mathscr{P}_{x}, E^{02}\right) \\
& \cong \operatorname{Hom}\left(\mathscr{P}_{x}, E^{10}\right), \text { by the Mukai spectral sequence for } E \\
& \cong \operatorname{Hom}\left(\Phi\left(\mathcal{O}_{x}\right), \Phi\left(E^{1}\right)\right) \\
& \cong \operatorname{Hom}\left(\mathcal{O}_{x}, E^{1}\right)
\end{aligned}
$$

Hence, as any map $\mathcal{O}_{x} \rightarrow E^{1}$ must factor through the torsion subsheaf of $E^{1}$ and $E^{1}$ is coherent, only finitely many of these maps can be non-zero. So $\operatorname{dim}\left\{x \in X: \operatorname{Ext}^{2}\left(\mathcal{O}_{x}, E^{0}\right) \neq 0\right\} \leqslant 0$. Therefore $E^{0}$ is a reflexive sheaf. 


\section{FMTS AND STABILITY CONDITIONS ON ABELIAN THREEFOLDS}

Proposition 4.6. Let $E \in \operatorname{Coh}(X)$. If $E \in \mathcal{T}_{0}$, then $E^{3}=0$, while if $E \in \mathcal{F}_{0}$, then $E^{0}=0$.

Proof. Let $E \in \mathcal{T}_{0}$. Then for any $x \in X$, we have

$$
\operatorname{Hom}\left(E^{3}, \mathcal{O}_{x}\right) \cong \operatorname{Hom}\left(\Phi(E)[3], \Phi\left(\mathscr{P}_{-x}\right)[3]\right) \cong \operatorname{Hom}\left(E, \mathscr{P}_{-x}\right)=0,
$$

as $\mathscr{P}_{-x} \in \mathcal{F}_{0}$. Therefore $E^{3}=0$, as required.

Now, let $E \in \mathcal{F}_{0}$. We may assume that $E$ is $\mu$-stable using $\mathrm{H}-\mathrm{N}$ and Jordan-Hölder filtrations. For generic $x \in X$ and $i=1,2$, we have

$$
\operatorname{Hom}\left(E^{1}, \mathcal{O}_{x}[i]\right)=\operatorname{Hom}\left(E^{2}, \mathcal{O}_{x}[i+1]\right)=\operatorname{Hom}\left(E^{3}, \mathcal{O}_{x}[i+2]\right)=0 .
$$

Hence for generic $x \in X$, we have

$$
\begin{aligned}
\operatorname{Hom}\left(E^{0}, \mathcal{O}_{x}\right) & \cong \operatorname{Hom}\left(\Phi(E), \mathcal{O}_{x}\right) \\
& \cong \operatorname{Hom}\left(\Phi(E), \Phi\left(\mathscr{P}_{-x}\right)[3]\right) \\
& \cong \operatorname{Hom}\left(E, \mathscr{P}_{-x}[3]\right) \\
& \cong \operatorname{Hom}\left(\mathscr{P}_{-x}, E\right)^{*}
\end{aligned}
$$

If $\mu(E)<0$, then $\operatorname{Hom}\left(\mathscr{P}_{-x}, E\right)=0$.

Assume $\mu(E)=0$. Since $E$ is assumed to be $\mu$-stable, any map in $\operatorname{Hom}\left(\mathscr{P}_{-x}, E\right)$ must be an isomorphism and so $E^{0}=0$. Consequently, for generic $x \in X$, we have $\operatorname{Hom}\left(E^{0}, \mathcal{O}_{x}\right)=0$. By Proposition 4.5 if $E^{0} \neq 0$, then it is reflexive. So $E^{0}=0$.

Proposition 4.7. Let $E \in \operatorname{Coh}(X)$; then $E^{3} \in \mathcal{T}_{0}$ and $E^{0} \in \mathcal{F}_{0}$.

Proof. For the first statement, let $T=T\left(E^{3}\right) \in \mathcal{T}_{0}$ and $F=F\left(E^{3}\right) \in \mathcal{F}_{0}$, so that

$$
0 \rightarrow T \rightarrow E^{3} \rightarrow F \rightarrow 0
$$

is a short exact sequence in $\operatorname{Coh}(X)$. We need to show $F=0$. Apply $\Phi$ to the short exact sequence above and consider the long exact sequence of $\operatorname{Coh}(X)$-cohomology. Then we have $F \in V_{\mathrm{Coh}(X)}^{\Phi}(1)$ and $T \in V_{\mathrm{Coh}(X)}^{\Phi}(0,1,2)$ (for the definition of $V$, see the notation section of the introduction), and

$$
0 \rightarrow T^{1} \rightarrow E^{31} \rightarrow F^{1} \rightarrow T^{2} \rightarrow 0
$$

is a long exact sequence in $\operatorname{Coh}(X)$. Here $E^{31} \cong E^{23}$ (from the Mukai spectral sequence (4.1) for $E$ ) and so

$$
\begin{aligned}
\operatorname{Hom}\left(E^{31}, F^{1}\right) & \cong \operatorname{Hom}\left(E^{23}, F^{1}\right) \\
& \cong \operatorname{Hom}\left(\Phi\left(E^{2}\right)[3], \Phi(F)[1]\right) \\
& \cong \operatorname{Hom}\left(E^{2}, F[-2]\right)=0 .
\end{aligned}
$$

Hence $F \cong(-1)^{*} F^{12} \cong(-1)^{*} T^{22}=0$ (from the Mukai spectral sequence (4.1) for $T$ ), as required.

The proof of the second statement is similar to that of the first.

Proposition 4.8. Let $E \in \mathcal{F}_{0}$. If $E^{1} \neq 0$, then $E^{1}$ is a reflexive sheaf.

Proof. By Proposition 4.6, we have $E^{0}=0$. Let $x \in X$. Then from the convergence of the Mukai spectral sequence (4.1) for $E$ and $0 \leqslant i \leqslant 2$, we have

$$
\begin{aligned}
\operatorname{Hom}\left(\mathcal{O}_{x}, E^{1}[i]\right) & \cong \operatorname{Hom}\left(\Phi\left(\mathcal{O}_{x}\right), \Phi\left(E^{1}\right)[i]\right) \\
& \cong \operatorname{Hom}\left(\mathscr{P}_{x}, E^{12}[i-2]\right)
\end{aligned}
$$




\section{A. Maciocia and D. Piyaratne}

as $\operatorname{Hom}\left(\mathscr{P}_{x}, \tau_{>2} \Phi\left(E^{1}\right)[i]\right) \cong \operatorname{Hom}\left(\mathscr{P}_{x}, E^{13}[i-3]\right)=0$. Hence $\operatorname{Hom}\left(\mathcal{O}_{x}, E^{1}\right)=\operatorname{Ext}^{1}\left(\mathcal{O}_{x}, E^{1}\right)=0$ and $\operatorname{Ext}^{2}\left(\mathcal{O}_{x}, E^{1}\right) \cong \operatorname{Hom}\left(\mathscr{P}_{x}, E^{12}\right)$.

From the convergence of the Mukai spectral sequence (4.1) for $E$,

$$
0 \rightarrow E^{20} \rightarrow E^{12} \rightarrow F \rightarrow 0
$$

is a short exact sequence in $\operatorname{Coh}(X)$. Here $F$ is a subobject of $(-1)^{*} E$. By applying the functor $\operatorname{Hom}\left(\mathscr{P}_{x},-\right)$, we obtain the exact sequence

$$
0 \rightarrow \operatorname{Hom}\left(\mathscr{P}_{x}, E^{20}\right) \rightarrow \operatorname{Hom}\left(\mathscr{P}_{x}, E^{12}\right) \rightarrow \operatorname{Hom}\left(\mathscr{P}_{x}, F\right) \rightarrow \cdots .
$$

Now $F \in \mathcal{F}_{0}$ and by Proposition 4.7, we also have $E^{20} \in \mathcal{F}_{0}$. Therefore we have $\operatorname{Hom}\left(\mathscr{P}_{x}, F\right) \neq 0$ or $\operatorname{Hom}\left(\mathscr{P}_{x}, E^{20}\right) \neq 0$ for at most a finite number of points $x \in X$; that is, $\operatorname{dim}\{x \in X$ : $\left.\operatorname{Ext}^{2}\left(\mathcal{O}_{x}, E^{1}\right) \neq 0\right\} \leqslant 0$. Hence $E^{1}$ is a reflexive sheaf.

Proposition 4.9. If $E$ is a torsion sheaf, then $E^{2} \in \mathcal{T}_{0}$.

Proof. Let $T:=T\left(E^{2}\right)$ and let $F:=F\left(E^{2}\right)$. Then $0 \rightarrow T \rightarrow E^{2} \rightarrow F \rightarrow 0$ is a short exact sequence in $\operatorname{Coh}(X)$. By applying $\Phi$, we obtain the long exact sequence

$$
0 \rightarrow T^{1} \rightarrow E^{21} \rightarrow F^{1} \rightarrow T^{2} \rightarrow 0
$$

in $\operatorname{Coh}(X)$. Here $F \in V_{\mathrm{Coh}(X)}^{\Phi}(1)$. From the convergence of the Mukai spectral sequence (4.1) for $E$, the sheaf $E^{21}$ fits into the $\operatorname{Coh}(X)$-short exact sequence

$$
0 \rightarrow Q \rightarrow E^{21} \rightarrow E^{13} \rightarrow 0
$$

where $Q$ is a quotient of $(-1)^{*} E$. So $Q$ is a torsion sheaf and $\operatorname{Hom}\left(Q, F^{1}\right)=0$, as $F^{1}$ is a reflexive sheaf (see Proposition 4.8). Therefore we have

$$
\begin{aligned}
\operatorname{Hom}\left(E^{21}, F^{1}\right) & \cong \operatorname{Hom}\left(E^{13}, F^{1}\right) \\
& \cong \operatorname{Hom}\left(\Phi\left(E^{1}\right)[3], \Phi(F)[1]\right) \\
& \cong \operatorname{Hom}\left(E^{1}, F[-2]\right)=0 .
\end{aligned}
$$

Hence $F^{1} \cong T^{2}$ and so $F \cong(-1)^{*} F^{12} \cong(-1)^{*} T^{22}=0$ (from the Mukai spectral sequence (4.1) for $T$ ), as required.

For $x \in X$, we denote $L \mathscr{P}_{x}$ by $L_{x}$. Since $h^{0}\left(X, L_{x}\right)=\chi\left(L_{x}\right)=1$, let the divisor $D_{x}$ be the zero locus of the unique (up to scaling) non-zero section $s_{x} \in H^{0}\left(X, L_{x}\right)$. Moreover, as $t_{x}^{*} L \otimes L^{-1}=\mathscr{P}_{x}$, we have $D_{x}=t_{x}^{*} D_{e}$, where $e \in X$ is the identity element. For a positive integer $m$, let $m D_{x}$ be the non-reduced divisor in the linear system $|m \ell|$ topologically supported on $D_{x}$. So $m D_{x}$ is the zero locus of the section $s_{x}^{\otimes m}$ of $L_{x}^{m}$, and we have the short exact sequence

$$
0 \rightarrow L_{x}^{-m} \rightarrow \mathcal{O}_{X} \rightarrow \mathcal{O}_{m D_{x}} \rightarrow 0
$$

in $\operatorname{Coh}(X)$. For $E \in \operatorname{Coh}(X)$, apply the functor $E \stackrel{\mathbf{L}}{\otimes}(-)$ to the short exact sequence above and consider the long exact sequence of $\operatorname{Coh}(X)$-cohomology. Since $L_{x}^{-m}$ and $\mathcal{O}_{X}$ are locally free, we have $\operatorname{Tor}_{i}\left(E, \mathcal{O}_{m D_{x}}\right)=0$ for $i \geqslant 2$. Now assume $E \in \operatorname{Coh}^{k}(X)$ for some $k \in\{0,1,2,3\}$. For generic $x \in X$, we have $\operatorname{dim}\left(\operatorname{Supp}(E) \cap D_{x}\right) \leqslant(k-1)$ and so $\operatorname{Tor}_{1}\left(E, \mathcal{O}_{m D_{x}}\right) \in \mathrm{Coh}^{\leqslant k-1}(X)$. However, $L_{x}^{-m} E \in \operatorname{Coh}^{k}(X)$, and so $\operatorname{Tor}_{1}\left(E, \mathcal{O}_{m D_{x}}\right)=0$. Therefore we have the short exact sequence

$$
\left.0 \rightarrow L_{x}^{-m} E \rightarrow E \rightarrow E\right|_{m D_{x}} \rightarrow 0
$$

in $\operatorname{Coh}(X)$. Since any $E \in \operatorname{Coh}(X)$ is an extension of sheaves from $\operatorname{Coh}^{k}(X)$, for generic $x \in X$, $\operatorname{Tor}_{i}\left(E, \mathcal{O}_{m D_{x}}\right)=0$ for $i \geqslant 1$ and so we have the short exact sequence (4.3) for all $E \in \operatorname{Coh}(X)$. 


\section{FMTS AND STABILITY CONDITIONS ON ABELIAN THREEFOLDS}

Moreover, when $0 \rightarrow E_{1} \rightarrow E_{2} \rightarrow E_{3} \rightarrow 0$ is a short exact sequence in $\operatorname{Coh}(X)$, for generic $x \in X$ we have $\operatorname{Tor}_{i}\left(E_{j}, \mathcal{O}_{m D_{x}}\right)=0$ for all $i \geqslant 1$ and each $j$, and so

$$
\left.\left.\left.0 \rightarrow E_{1}\right|_{m D_{x}} \rightarrow E_{2}\right|_{m D_{x}} \rightarrow E_{3}\right|_{m D_{x}} \rightarrow 0
$$

is a short exact sequence in $\operatorname{Coh}(X)$.

Proposition 4.10. Let $E \in \operatorname{Coh}^{\leqslant 1}(X)$; then $E^{1} \in \mathcal{T}_{0}$.

Proof. The sheaf $E \in \mathrm{Coh}^{\leqslant 1}(X)$ fits into the torsion sequence $0 \rightarrow E_{0} \rightarrow E \rightarrow E_{1} \rightarrow 0$, where $E_{0} \in \operatorname{Coh}^{0}(X)$ and $E_{1} \in \operatorname{Coh}^{1}(X)$. Here $E_{0} \in V_{\operatorname{Coh}(X)}^{\Phi}(0)$ and so $E^{1}=E_{1}^{1}$. Therefore we only need to prove the claim for a pure dimension one torsion sheaf $E$. Then for sufficiently large $m>0$ and suitable $x \in X$, we have $L_{x}^{-m} E \in V_{\mathrm{Coh}(X)}^{\Phi}(1)$, and

$$
\left.0 \rightarrow L_{x}^{-m} E \rightarrow E \rightarrow E\right|_{m D_{x}} \rightarrow 0
$$

is a short exact sequence in $\operatorname{Coh}(X)$ for $\left.E\right|_{m D_{x}} \in \operatorname{Coh}^{0}(X)$. By applying the Fourier-Mukai transform $\Phi$, we have $\left(L_{x}^{-m} E\right)^{1} \rightarrow E^{1}$. Therefore, we only need to show $\left(L_{x}^{-m} E\right)^{1} \in \mathcal{T}_{0}$. Let us show this by proving the claim for a pure dimension one torsion sheaf $E \in V_{\mathrm{Coh}(X)}^{\Phi}(1)$. Then $\operatorname{ch}(E)=(0,0, \alpha, \beta)$, where $\alpha>0$ and $\beta \leqslant 0$ since $\beta=-\operatorname{rk}\left(E^{1}\right)$.

Let $T:=T\left(E^{1}\right)$ and let $F:=F\left(E^{1}\right)$. Then $0 \rightarrow T \rightarrow E^{1} \rightarrow F \rightarrow 0$ is a short exact sequence in $\operatorname{Coh}(X)$. We need to show $F=0$. Suppose $F \neq 0$ for a contradiction. Apply the FourierMukai transform $\Phi$ and consider the long exact sequence of $\operatorname{Coh}(X)$-cohomology. Then we have $T \in V_{\mathrm{Coh}(X)}^{\Phi}(2)$ and $F \in V_{\mathrm{Coh}(X)}^{\Phi}(1,2)$, and

$$
0 \rightarrow F^{1} \rightarrow T^{2} \rightarrow E \rightarrow F^{2} \rightarrow 0
$$

is a long exact sequence in $\operatorname{Coh}(X)$.

(i) Assume that the map $T^{2} \rightarrow E$ is zero. Then $T \cong(-1)^{*} T^{21} \cong(-1)^{*} F^{11}=0$ from the Mukai spectral sequence (4.1), as $F \in V_{\mathrm{Coh}(X)}^{\Phi}(1,2)$. So $E=F^{2}$ and hence $F \in V_{\mathrm{Coh}(X)}^{\Phi}(2)$. Therefore $F \cong(-1)^{*} E^{1}$ and $\operatorname{so} \operatorname{ch}(F)=(-\beta, \alpha, 0,0)$. Here $\alpha>0$, which is not possible as $\mu(F) \leqslant 0$.

(ii) Now assume that the map $T^{2} \rightarrow E$ is non-zero. Let $K=\operatorname{im}\left(T^{2} \rightarrow E\right)$. Then $K \in \operatorname{Coh}^{1}(X)$ and the $\operatorname{Coh}(X)$-short exact sequence $0 \rightarrow F^{1} \rightarrow T^{2} \rightarrow K \rightarrow 0$ corresponds to an element from $\operatorname{Ext}^{1}\left(K, F^{1}\right)$. Here $F^{1}$ is a reflexive sheaf and so there exist a locally free sheaf $U$ and a torsion free sheaf $V$ such that $0 \rightarrow F^{1} \rightarrow U \rightarrow V \rightarrow 0$ is a non-splitting short exact sequence in $\operatorname{Coh}(X)$. By applying the functor $\operatorname{Hom}(K,-)$, we obtain the following exact sequence:

$$
\cdots \rightarrow \operatorname{Hom}(K, V) \rightarrow \operatorname{Ext}^{1}\left(K, F^{1}\right) \rightarrow \operatorname{Ext}^{1}(K, U) \rightarrow \cdots
$$

Here $\operatorname{Hom}(K, V)=0$ and $\operatorname{Ext}^{1}(K, U) \cong \operatorname{Ext}^{2}(U, K)^{*} \cong H^{2}\left(X, U^{*} \otimes K\right)^{*}=0$, as $K \in \operatorname{Coh}^{\leqslant 1}(X)$. So $\operatorname{Ext}^{1}\left(K, F^{1}\right)=0$ implies $T^{2} \cong F^{1} \oplus K$. Here $T^{2} \in V_{\mathrm{Coh}(X)}^{\Phi}(1)$ implies $F^{1}=0$ and so $K \cong T^{2}$. Then $F^{2} \cong E / T^{2}$ and also $F \in V_{\operatorname{Coh}(X)}^{\Phi}(2)$. Since $F^{2}$ is an element of $V_{\operatorname{Coh}(X)}^{\Phi}(1)$, it is a pure dimension one torsion sheaf. So $\operatorname{ch}\left(F^{2}\right)=\left(0,0, \alpha^{\prime}, \beta^{\prime}\right)$, where $\alpha^{\prime}>0$ and $\beta^{\prime} \leqslant 0$. Therefore $\operatorname{ch}(F)=\left(-\beta^{\prime}, \alpha^{\prime}, 0,0\right)$, which is not possible as $\mu(F) \leqslant 0$ implies $\alpha^{\prime} \leqslant 0$.

Therefore $F=0$, as required to complete the proof.

Recall from [Muk78, Proposition 6.16] that for any positive integer $s$, the semi-homogeneous bundle $\left(L^{s}\right)^{0}$ is slope stable. In the rest of this section we abuse notation and write $\left(L^{s}\right)^{0}$ for the functor $\left(L^{s}\right)^{0} \otimes-$. 


\section{A. Maciocia and D. Piyaratne}

Proposition 4.11. Let $E_{n} \in \mathrm{HN}[0,+\infty)$ for $n \in \mathbb{N}$ be a sequence of coherent sheaves on $X$. Suppose that for any $s>0$, there is an $N(s)>0$ such that for any $n>N(s)$, we have $\left(L^{s}\right)^{0} E_{n} \in V_{\mathrm{Coh}(X)}^{\Phi}(3)$. Then $\mu^{+}\left(E_{n}\right) \rightarrow 0$ as $n \rightarrow+\infty$.

Proof. Assume $\mu^{+}\left(E_{n}\right) \not \rightarrow 0$ as $n \rightarrow+\infty$ for a contradiction. Then there exists an $\varepsilon>0$ such that for any $N^{\prime}>0$ there is an $n>N^{\prime}$ satisfying $\mu^{+}\left(E_{n}\right)>\varepsilon$.

Let $T_{n}$ be the slope-semistable $\mathrm{H}-\mathrm{N}$ factor of $E_{n}$ with the highest slope; that is, $\mu\left(T_{n}\right)=$ $\mu^{+}\left(E_{n}\right)$. There is an $s \in \mathbb{N}$ such that $\mu\left(\left(L^{s}\right)^{0}\right)>-\varepsilon$. Then for any $N^{\prime}>0$, there is an $n>N^{\prime}$ such that $\left(L^{s}\right)^{0} T_{n} \in \mathcal{T}_{0}$. Consequently, for some $n>N(s)$ we have

$$
\operatorname{Hom}\left(\left(L^{s}\right)^{0} T_{n},\left(L^{s}\right)^{0} E_{n}\right) \cong \operatorname{Hom}\left(\Phi\left(\left(L^{s}\right)^{0} T_{n}\right), \Phi\left(\left(L^{s}\right)^{0} E_{n}\right)\right) \cong 0,
$$

as $\left(\left(L^{s}\right)^{0} T_{n}\right)^{3}=0$ (from Proposition 4.6) and $\left(L^{s}\right)^{0} E_{n} \in V_{\mathrm{Coh}(X)}^{\Phi}(3)$. This is the contradiction required to complete the proof.

Let $s$ be a positive integer. Consider the Fourier-Mukai functor defined by

$$
\Pi=\Phi \circ\left(L^{s}\right)^{0} \circ \Phi[3] .
$$

Then $\Pi_{\operatorname{Coh}(X)}^{i}\left(\mathcal{O}_{x}\right)=0$ for $i \neq 0$ and $\Pi_{\operatorname{Coh}(X)}^{0}\left(\mathcal{O}_{x}\right)=L^{s} \mathscr{P}_{y}$ for some $y \in X$. Define the FourierMukai functor

$$
\widehat{\Pi}=\Phi \circ\left(L^{-s}\right)^{3} \circ \Phi .
$$

One can show that $\widehat{\Pi}[3]$ is right and left adjoint to $\Pi$ (and vice versa). We have $\widehat{\Pi}_{\operatorname{Coh}(X)}^{i}\left(\mathcal{O}_{x}\right)=0$ for $i \neq 0$, and $\widehat{\Pi}_{\operatorname{Coh}(X)}^{0}\left(\mathcal{O}_{x}\right)=L^{-s} \mathscr{P}_{z}$ for some $z \in X$. Therefore $\Pi$ is a Fourier-Mukai functor with kernel a locally free sheaf $\mathcal{U}$ on $X \times X$.

We have the spectral sequence

$$
\Phi^{p}\left(\left(L^{s}\right)^{0} \Phi^{q}(E)\right) \Longrightarrow \Pi^{p+q-3}(E)
$$

for $E$.

Proposition 4.12. Let $E$ be a coherent sheaf such that $L^{-n} E \in V_{\mathrm{Coh}(X)}^{\Phi}(k)$ for sufficiently large $n$, where $k \in\{0, \ldots, 3\}$. Then $\mu^{+}\left(\left(L^{-n} E\right)^{k}\right) \rightarrow 0$ as $n \rightarrow+\infty$.

Proof. Since $L^{-n} E \in V_{\mathrm{Coh}(X)}^{\Phi}(k)$ for sufficiently large $n$, we have $E \in \operatorname{Coh}^{k}(X)$. If $k=0$, then $E \in \operatorname{Coh}^{0}(X)$ and so we have $\mu^{+}\left(\left(L^{-n} E\right)^{0}\right)=0$. Otherwise, by Propositions 4.10, 4.9 and 4.7, for $E \in \operatorname{Coh}^{k}(X)$ we have $\left(L^{-n} E\right)^{k} \in \mathcal{T}_{0}$. Let $s$ be a positive integer. Consider the convergence of the spectral sequence (4.4). For large enough $n$, we also have $L^{-n} E \in V_{\operatorname{Coh}(X)}^{\Pi}(k)$. Therefore $\left(L^{s}\right)^{0}\left(L^{-n} E\right)^{k} \in V_{\operatorname{Coh}(X)}^{\Phi}(3)$. By Proposition 4.11, we have $\mu^{+}\left(\left(L^{-n} E\right)^{k}\right) \rightarrow 0$ as $n \rightarrow+\infty$.

Proposition 4.13. Let $E$ be a reflexive sheaf. Then for sufficiently large $n>0, L^{-n} E \in$ $V_{\mathrm{Coh}(X)}^{\Phi}(2,3)$, and we have $\left(L^{-n} E\right)^{2} \cong\left(T_{0}\right)^{0}$ for some $T_{0} \in \mathrm{Coh}^{0}(X)$.

Proof. Consider a minimal locally free resolution of $E$,

$$
0 \rightarrow F_{2} \rightarrow F_{1} \rightarrow E \rightarrow 0
$$

By applying the Fourier-Mukai transform $\Phi L^{-n}$ for sufficiently large $n>0$, we obtain $L^{-n} E \in$ $V_{\mathrm{Coh}(X)}^{\Phi}(2,3)$.

Since $E$ is a reflexive sheaf, there are a locally free sheaf $P$ and a torsion-free sheaf $Q$ such that

$$
0 \rightarrow E \rightarrow P \rightarrow Q \rightarrow 0
$$




\section{FMTS AND STABILITY CONDITIONS ON ABELIAN THREEFOLDS}

is a short exact sequence in $\operatorname{Coh}(X)$. By applying the Fourier-Mukai transform $\Phi L^{-n}$ for sufficiently large $n$, we have $\left(L^{-n} E\right)^{2} \cong\left(L^{-n} Q\right)^{1}$.

The torsion-free sheaf $Q$ fits into the short exact sequence $0 \rightarrow Q \rightarrow Q^{* *} \rightarrow T \rightarrow 0$ for some $T \in \mathrm{Coh}^{\leqslant 1}(X)$. Apply the Fourier-Mukai transform $\Phi L^{-n}$ for sufficiently large $n$ and consider the long exact sequence of $\operatorname{Coh}(X)$-cohomology. Since $L^{-n} Q^{* *} \in V_{\operatorname{Coh}(X)}^{\Phi}(2,3)$, we have $\left(L^{-n} Q\right)^{1} \cong\left(L^{-n} T\right)^{0}$. The torsion sheaf $T \in \mathrm{Coh}^{\leqslant 1}(X)$ fits into a short exact sequence $0 \rightarrow T_{0} \rightarrow$ $T \rightarrow T_{1} \rightarrow 0$ in $\operatorname{Coh}(X)$ for $T_{i} \in \operatorname{Coh}^{i}(X)$ with $i=0,1$. Therefore $\left(L^{-n} T\right)^{0} \cong\left(T_{0}\right)^{0}$, and so $\left(L^{-n} E\right)^{2} \cong\left(T_{0}\right)^{0}$, as required.

Proposition 4.14. Let $E \in \operatorname{Coh}^{1}(X)$ with $E \in V_{\mathrm{Coh}(X)}^{\Phi}(1)$. If $0 \neq T \in \mathrm{HN}[0,+\infty]$ is a subsheaf of $E^{1}$, then $\ell \operatorname{ch}_{2}(T) \leqslant 0$.

Proof. For $n>0$ and generic $z \in X$, we have the $\operatorname{Coh}(X)$-short exact sequence

$$
0 \rightarrow L_{z}^{-n} E \rightarrow E \rightarrow T_{0} \rightarrow 0
$$

for $T_{0}:=\left.E\right|_{n D_{z}} \in \operatorname{Coh}^{0}(X)$. By applying the Fourier-Mukai transform $\Phi$, we get the commutative diagram

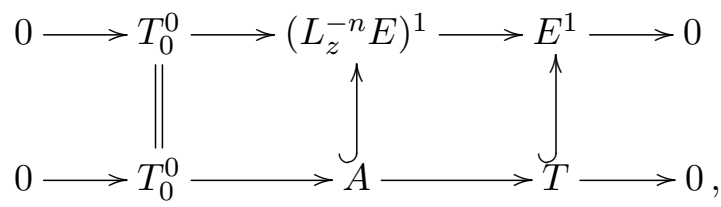

for some $A \in \mathrm{HN}[0,+\infty]$. So we have $\operatorname{ch}_{k}(A)=\operatorname{ch}_{k}(T)$ for $k=1,2,3$.

Let $G$ be a slope-semistable $\mathrm{H}-\mathrm{N}$ factor of $A$. From the usual B-G inequality, we have $\ell\left(\operatorname{ch}_{1}(G)^{2}-2 \operatorname{ch}_{0}(G) \operatorname{ch}_{2}(G)\right) \geqslant 0$. Consequently,

$$
\begin{aligned}
2 \ell \operatorname{ch}_{2}(G) & \leqslant \frac{\ell \operatorname{ch}_{1}(G)^{2}}{\operatorname{ch}_{0}(G)} \\
& =\ell^{2} \operatorname{ch}_{1}(G) \mu(G) \\
& \leqslant \ell^{2} \operatorname{ch}_{1}(A) \mu(G) \\
& \leqslant \ell^{2} \operatorname{ch}_{1}(T) \mu^{+}\left(\left(L_{z}^{-n} E\right)^{1}\right) .
\end{aligned}
$$

By Proposition 4.12, we have $\mu^{+}\left(\left(L_{z}^{-n} E\right)^{1}\right) \rightarrow 0$ as $n \rightarrow+\infty$. So choose $n>0$ large enough such that $\ell^{2} \operatorname{ch}_{1}(T) \mu^{+}\left(\left(L_{z}^{-n} E\right)^{1}\right)<\ell^{3}$. Since $2 \ell \operatorname{ch}_{2}(G) \in \ell^{3} \mathbb{Z}$, we have $\ell \operatorname{ch}_{2}(G) \leqslant 0$. So $\ell \operatorname{ch}_{2}(T)=$ $\ell \operatorname{ch}_{2}(A) \leqslant 0$.

Proposition 4.15. (i) Let $E \in \mathcal{F}_{0}$ be a reflexive sheaf. If $0 \neq T \in \mathcal{T}_{0}$ is a subsheaf of $E^{1}$, then $\ell \operatorname{ch}_{2}(T) \leqslant 0$.

(ii) Let $E \in \mathcal{T}_{0}$ be a torsion free. If $0 \neq F \in \mathcal{F}_{0}$ is a quotient of $E^{2}$, then $\ell \operatorname{ch}_{2}(F) \leqslant 0$.

Proof. (i) Recall that, for any positive integer $m$, non-reduced divisors $m D_{x}$ of $L_{x}^{m}$ are topologically supported on $D_{x}$.

Since $E$ is a reflexive sheaf, one can choose $x, y \in X$ such that

$-\operatorname{dim}\left(D_{x} \cap D_{y}\right)=1$;

- the restriction $\left.E\right|_{D_{x}}$ is locally free on $D_{x}$; and

- the restriction $\left.E\right|_{D_{y}}$ is locally free on $D_{y}$. 


\section{A. Maciocia and D. Piyaratne}

By Proposition 4.13, for sufficiently large $m>0$, we have $L_{x}^{-m} E \in V_{\mathrm{Coh}(X)}^{\Phi}(2,3)$. By applying the Fourier-Mukai transform $\Phi$ to the $\operatorname{Coh}(X)$-short exact sequence

$$
\left.0 \rightarrow L_{x}^{-m} E \rightarrow E \rightarrow E\right|_{m D_{x}} \rightarrow 0
$$

we obtain $\left.E\right|_{m D_{x}} \in V_{\mathrm{Coh}(X)}^{\Phi}(1,2)$ and $E^{1} \hookrightarrow\left(\left.E\right|_{m D_{x}}\right)^{1}$. Since $\left.E\right|_{D_{x}}$ is locally free on $D_{x}$, for large enough $n>0$, we have $\left.L_{y}^{-n} E\right|_{m D_{x}} \in V_{\mathrm{Coh}(X)}^{\Phi}(2)$. By applying the Fourier-Mukai transform $\Phi$ to the $\operatorname{Coh}(X)$-short exact sequence

$$
\left.\left.\left.0 \rightarrow L_{y}^{-n} E\right|_{m D_{x}} \rightarrow E\right|_{m D_{x}} \rightarrow E\right|_{m D_{x} \cap n D_{y}} \rightarrow 0,
$$

we obtain $\left.E\right|_{m D_{x} \cap n D_{y}} \in V_{\operatorname{Coh}(X)}^{\Phi}(1)$ and $\left(\left.E\right|_{m D_{x}}\right)^{1} \hookrightarrow\left(\left.E\right|_{m D_{x} \cap n D_{y}}\right)^{1}$. Therefore we have

$$
T \hookrightarrow E^{1} \hookrightarrow\left(\left.E\right|_{m D_{x} \cap n D_{y}}\right)^{1} .
$$

The result follows from Proposition 4.14.

(ii) Since $F \neq 0$ is a quotient of $E^{2}$, we have $F^{*} \hookrightarrow\left(E^{2}\right)^{*}$. Here $F^{*} \in \mathrm{HN}[0,+\infty)$ fits into the $\mathrm{Coh}(X)$-short exact sequence $0 \rightarrow T \rightarrow F^{*} \rightarrow F_{0} \rightarrow 0$ for some $T \in \mathcal{T}_{0}$ and $F_{0} \in \mathrm{HN}[0]$. By the usual B-G inequality, $\ell \operatorname{ch}_{2}\left(F_{0}\right) \leqslant 0$.

By Proposition 4.6, we have $E^{3}=0=\left(E^{*}\right)^{0}$. Therefore from the convergence of the duality spectral sequence (4.2) for $E$, we have the $\operatorname{Coh}(X)$-short exact sequence

$$
0 \rightarrow(-1)^{*}\left(E^{*}\right)^{1} \rightarrow\left(E^{2}\right)^{*} \rightarrow P \rightarrow 0
$$

for some subsheaf $P$ of $\left(\mathcal{E} x t^{1}(E, \mathcal{O})\right)^{0}$. By Proposition 4.7, we have $\left(\mathcal{E} x t^{1}(E, \mathcal{O})\right)^{0} \in \mathcal{F}_{0}$ and so $P \in \mathcal{F}_{0}$. Therefore $\operatorname{Hom}(T, P)=0$ and so $P \hookrightarrow(-1)^{*}\left(E^{*}\right)^{1}$. Here $E^{*} \in \mathcal{F}_{0}$ and so by part (i), we have $\ell \operatorname{ch}_{2}(T) \leqslant 0$. Therefore $\ell \operatorname{ch}_{2}(F) \leqslant \ell \operatorname{ch}_{2}\left(F^{* *}\right)=\ell \operatorname{ch}_{2}\left(F^{*}\right)=\ell \operatorname{ch}_{2}\left(F_{0}\right)+\ell \operatorname{ch}_{2}(T) \leqslant 0$.

Proposition 4.16. Let $E \in \operatorname{Coh}(X)$.

(i) If $E \in \mathcal{F}_{0}$, then $E^{1} \in \mathcal{F}_{0}$.

(ii) If $E \in \operatorname{HN}[0,+\infty)$ with $E^{3}=0$, then $E^{2} \in \operatorname{HN}[0,+\infty]$.

Proof. (i) Assume the opposite for a contradiction. Let $T:=T\left(E^{1}\right)$ and $F:=F\left(E^{1}\right)$. Then $0 \rightarrow T \rightarrow E^{1} \rightarrow F \rightarrow 0$ is a short exact sequence in $\operatorname{Coh}(X)$. By Proposition 4.8 , the object $E^{1}$ is reflexive and so non-trivial $T$ is reflexive. Hence $\ell^{2} \operatorname{ch}_{1}(T)>0$. By applying the FourierMukai transform $\Phi$ to this short exact sequence we obtain $T \in V_{\operatorname{Coh}(X)}^{\Phi}(2)$ and $F \in V_{\operatorname{Coh}(X)}^{\Phi}(1,2)$. Moreover, we have the $\operatorname{Coh}(X)$-short exact sequence

$$
0 \rightarrow F^{1} \rightarrow T^{2} \rightarrow E_{1} \rightarrow 0
$$

for some subsheaf $E_{1}$ of $E^{12}$. From the Mukai spectral sequence (4.1) for $E$ we have the $\operatorname{Coh}(X)$ short exact sequence

$$
0 \rightarrow E^{20} \rightarrow E^{12} \rightarrow E_{2} \rightarrow 0
$$

for some subsheaf $E_{2}$ of $(-1)^{*} E$. Therefore $E_{2} \in \mathcal{F}_{0}$ and by Proposition 4.7, we have $E^{20} \in \mathcal{F}_{0}$. So we have $E^{12} \in \mathcal{F}_{0}$, and consequently $E_{1} \in \mathcal{F}_{0}$.

Let $T_{1}:=T\left(F^{1}\right)$ and $F_{1}:=F\left(T^{2}\right)$. These fit into the following commutative diagram for 
some $F_{2} \in \mathcal{F}_{0}$ :

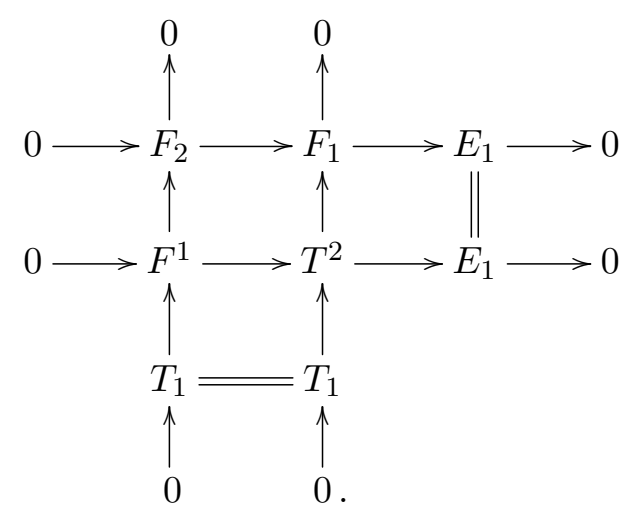

By Proposition 4.15, we have $\ell \operatorname{ch}_{2}\left(F_{1}\right) \leqslant 0$.

By applying the Fourier-Mukai transform $\Phi$ to the $\operatorname{Coh}(X)$-short exact sequence $0 \rightarrow T_{1} \rightarrow$ $F^{1} \rightarrow F_{2} \rightarrow 0$, we obtain the $\operatorname{Coh}(X)$-short exact sequence

$$
0 \rightarrow F_{2}^{1} \rightarrow T_{1}^{2} \rightarrow F_{3} \rightarrow 0
$$

for some subsheaf $F_{3}$ of $F^{12}$. We also have $T_{1} \in V_{\mathrm{Coh}(X)}^{\Phi}(2)$. By considering the Mukai spectral sequence (4.1) for $F$, one can show $F_{3} \in \mathcal{F}_{0}$. By Proposition 4.8, the sheaf $F_{2}^{1}$ is reflexive. So $T_{1}^{2}$ is torsion free and fits into the $\operatorname{Coh}(X)$-short exact sequence

$$
0 \rightarrow T_{1}^{2} \rightarrow\left(T_{1}^{2}\right)^{* *} \rightarrow Q \rightarrow 0
$$

for some $Q \in \operatorname{Coh}^{\leqslant 1}(X)$. The torsion sheaf $Q$ fits into the $\operatorname{Coh}(X)$-short exact sequence

$$
0 \rightarrow Q_{0} \rightarrow Q \rightarrow Q_{1} \rightarrow 0
$$

for $Q_{0} \in \operatorname{Coh}^{0}(X)$ and $Q_{1} \in \operatorname{Coh}^{1}(X)$. By Proposition 4.13, for large enough $m>0$, we have $\left(L^{-m} T_{1}^{2}\right)^{1} \cong\left(L^{-m} Q\right)^{0} \cong Q_{0}^{0}$. Also, $\left(L^{-m} Q_{1}\right)^{1} \cong\left(L^{-m} Q\right)^{1}$ and $\left(L^{-m}\left(T_{1}^{2}\right)^{* *}\right)^{2} \cong R_{0}^{0}$ for some $R_{0} \in \operatorname{Coh}^{0}(X)$. So we have the $\operatorname{Coh}(X)$-short exact sequence

$$
0 \rightarrow\left(L^{-m} Q_{1}\right)^{1} \rightarrow\left(L^{-m} T_{1}^{2}\right)^{2} \rightarrow R_{0}^{0} \rightarrow 0 .
$$

By Proposition 4.10, we have $\left(L^{-m} T_{1}^{2}\right)^{2} \in \mathrm{HN}[0,+\infty)$ and $\ell \operatorname{ch}_{2}\left(\left(L^{-m} T_{1}^{2}\right)^{2}\right)=0$.

The torsion-free sheaf $F_{3}$ also fits into the $\operatorname{Coh}(X)$-short exact sequence $0 \rightarrow F_{3} \rightarrow F_{3}^{* *} \rightarrow$ $S \rightarrow 0$ for some $S \in \mathrm{Coh}^{\leqslant 1}(X)$.

Choose $x, y \in X$ such that

$-\operatorname{dim}\left(D_{x} \cap D_{y}\right)=1$,

- $D_{x} \cap \operatorname{Supp}\left(Q_{0}\right)=\emptyset$,

$-\operatorname{dim}\left(\operatorname{Supp}\left(Q_{1}\right) \cap D_{x}\right) \leqslant 0$,

- $D_{x} \cap D_{y} \cap \operatorname{Supp}(Q)=\emptyset$,

- $D_{x} \cap D_{y} \cap \operatorname{Supp}(S)=\emptyset$.

Since $F_{2}^{1}$ is reflexive, $\left.F_{2}^{1}\right|_{D_{x}}$ is locally free on $D_{x}$ and $\left.F_{2}^{1}\right|_{D_{y}}$ is locally free on $D_{y}$, and since $F_{3}^{* *}$ is reflexive, $\left.F_{3}^{* *}\right|_{D_{x}}$ is locally free on $D_{x}$ and $\left.F_{3}^{* *}\right|_{D_{y}}$ is locally free on $D_{y}$.

From the Mukai spectral sequence for $F_{2}$, we know $F_{2}^{1} \in V_{\operatorname{Coh}(X)}^{\Phi}(2,3)$. Since it is reflexive, for large enough $m>0$, the sheaf $L_{x}^{-m} F_{2}^{1}$ is an element of $V_{\mathrm{Coh}(X)}^{\Phi}(2,3)$, and since $\left.F_{2}^{1}\right|_{D_{x}}$ is locally free on $D_{x}$, we have $\left.L_{y}^{-n} F_{2}^{1}\right|_{m D_{x}} \in V_{\operatorname{Coh}(X)}^{\Phi}(2)$. So $\left.F_{2}^{1}\right|_{m D_{x} \cap n D_{y}} \in V_{\operatorname{Coh}(X)}^{\Phi}(1)$. Since 


\section{A. Maciocia and D. Piyaratne}

$D_{x} \cap D_{y} \cap \operatorname{Supp}(S)=\emptyset$, similarly one can show $\left.\left.F_{3}\right|_{m D_{x} \cap n D_{y}} \cong F_{3}^{* *}\right|_{m D_{x} \cap n D_{y}}$ is in $V_{\mathrm{Coh}(X)}^{\Phi}(1)$. Therefore, $\left.\left.T_{1}^{2}\right|_{m D_{x} \cap n D_{y}} \cong\left(T_{1}^{2}\right)^{* *}\right|_{m D_{x} \cap n D_{y}}$ is in $V_{\mathrm{Coh}(X)}^{\Phi}(1)$.

By applying the Fourier-Mukai transform $\Phi$ to the $\operatorname{Coh}(X)$-short exact sequence

$$
\left.0 \rightarrow L_{x}^{-m} T_{1}^{2} \rightarrow T_{1}^{2} \rightarrow T_{1}^{2}\right|_{m D_{x}} \rightarrow 0
$$

for large enough $m>0$ we obtain the $\operatorname{Coh}(X)$-long exact sequence

$$
0 \rightarrow Q_{0}^{0} \rightarrow(-1)^{*} T_{1} \rightarrow\left(\left.T_{1}^{2}\right|_{m D_{x}}\right)^{1} \rightarrow\left(L^{-m} T_{1}^{2}\right)^{2} \rightarrow 0 .
$$

So $\left(\left.T_{1}^{2}\right|_{m D_{x}}\right)^{1} \in \mathrm{HN}[0,+\infty]$ and $\operatorname{ch}_{2}\left(\left(\left.T_{1}^{2}\right|_{m D_{x}}\right)^{1}\right)=\operatorname{ch}_{2}\left(T_{1}\right)$. Moreover, we have the $\operatorname{Coh}(X)$-short exact sequence

$$
\left.\left.\left.0 \rightarrow T_{1}^{2}\right|_{m D_{x}} \rightarrow\left(T_{1}^{2}\right)^{* *}\right|_{m D_{x}} \rightarrow Q_{1}\right|_{m D_{x}} \rightarrow 0 .
$$

Here $\left.Q_{1}\right|_{m D_{x}} \in \operatorname{Coh}^{0}(X)$. So for large enough $n>0$, we have $\left(\left.L^{-n} T_{1}^{2}\right|_{m D_{x}}\right)^{1} \cong\left(\left.Q_{1}\right|_{m D_{x}}\right)^{0}$.

By applying the Fourier-Mukai transform $\Phi$ to the $\operatorname{Coh}(X)$-short exact sequence

$$
\left.\left.\left.0 \rightarrow L_{y}^{-n} T_{1}^{2}\right|_{m D_{x}} \rightarrow T_{1}^{2}\right|_{m D_{x}} \rightarrow T_{1}^{2}\right|_{m D_{x} \cap n D_{y}} \rightarrow 0,
$$

we have the $\operatorname{Coh}(X)$-long exact sequence

$$
0 \rightarrow\left(\left.Q_{1}\right|_{m D_{x}}\right)^{0} \stackrel{\alpha}{\rightarrow}\left(\left.T_{1}^{2}\right|_{m D_{x}}\right)^{1} \rightarrow\left(\left.T_{1}^{2}\right|_{m D_{x} \cap n D_{y}}\right)^{1} \rightarrow \cdots .
$$

Let $T_{2}:=\operatorname{coker}(\alpha)$. Then $T_{2} \in \operatorname{HN}[0,+\infty]$ and $\operatorname{ch}_{2}\left(T_{2}\right)=\operatorname{ch}_{2}\left(T_{1}\right)$. By Proposition 4.14, we have $\ell \operatorname{ch}_{2}\left(T_{2}\right) \leqslant 0$. So $\ell \operatorname{ch}_{2}\left(T^{2}\right)=\ell \operatorname{ch}_{2}\left(T_{1}\right)+\ell \operatorname{ch}_{2}\left(F_{1}\right) \leqslant 0$. Therefore we have $\ell^{2} \operatorname{ch}_{1}(T) \leqslant 0$. This is the contradiction required to complete the proof.

(ii) Since $E^{*} \in \mathrm{HN}(-\infty, 0]$, it follows from part (i) that $\left(E^{*}\right)^{1} \in \mathrm{HN}(-\infty, 0]$. By the convergence of the duality spectral sequence (4.2) for $E$, we have $\left(E^{2}\right)^{*} \in \operatorname{HN}(-\infty, 0]$. So $E^{2} \in \mathrm{HN}[0,+\infty]$, as required.

Corollary 4.17. Let $E \in \mathcal{T}_{0}$; then $E^{2} \in \mathcal{T}_{0}$.

Proof. Let $T:=T\left(E^{2}\right)$ and $F:=F\left(E^{2}\right)$. Then $0 \rightarrow T \rightarrow E^{2} \rightarrow F \rightarrow 0$ is a short exact sequence in $\operatorname{Coh}(X)$. Now we need to show $F=0$. Apply the Fourier-Mukai transform $\Phi$ and consider the long exact sequence of $\operatorname{Coh}(X)$-cohomology. So we have $F \in V_{\operatorname{Coh}(X)}^{\Phi}(1)$ and

$$
0 \rightarrow T^{1} \rightarrow E^{21} \rightarrow F^{1} \rightarrow T^{2} \rightarrow 0
$$

is a long exact sequence in $\operatorname{Coh}(X)$. From the convergence of the Mukai spectral sequence (4.1) for $E$, we have the $\operatorname{Coh}(X)$-short exact sequence

$$
0 \rightarrow Q \rightarrow E^{21} \rightarrow E^{13} \rightarrow 0,
$$

where $Q$ is a quotient of $(-1)^{*} E$. Then $Q \in \mathcal{T}_{0}$ and, by Proposition 4.7, we have $E^{13} \in \mathcal{T}_{0}$ and so $E^{21} \in \mathcal{T}_{0}$. On the other hand, by Proposition 4.16 , we have $F^{1} \in \mathcal{F}_{0}$. So the map $E^{21} \rightarrow F^{1}$ is zero and $F^{1} \cong T^{2}$. Hence $F \cong(-1)^{*} F^{12} \cong(-1)^{*} T^{22}=0$ (from the Mukai spectral sequence (4.1) for $T$ ), as required.

Proposition 4.18. Let $E \in \operatorname{HN}(0,1]$; then $E^{0} \in \mathrm{HN}\left(-\infty,-\frac{1}{2}\right]$.

Proof. Due to Mukai, we know that $\Phi L \Phi \cong(-1)^{*} L^{-1} \Phi L^{-1}$. Therefore we have the following convergence of a spectral sequence:

$$
E_{2}^{p, q}=\Phi_{\operatorname{Coh}(X)}^{p} L \Phi_{\operatorname{Coh}(X)}^{q}(E) \Longrightarrow(-1)^{*} L^{-1} \Phi_{\operatorname{Coh}(X)}^{p+q}\left(L^{-1} E\right) .
$$




\section{FMTS AND STABILITY CONDITIONS ON ABELIAN THREEFOLDS}

Here $L^{-1} E \in \mathrm{HN}(-1,0]$, and so by Proposition 4.6, we have $\left(L^{-1} E\right)^{0}=0$. So from the convergence of the spectral sequence above for $E$ we have $\left(L E^{0}\right)^{0}=0$. Also, $\left(L E^{0}\right)^{1} \hookrightarrow L^{-1}\left(L^{-1} E\right)^{1}$. By Proposition 4.16, we have $\left(L^{-1} E\right)^{1} \in \mathcal{F}_{0}$ and so $\left(L E^{0}\right)^{1} \in \operatorname{HN}(-\infty,-1] \subset \mathcal{F}_{0}$.

Let $F \subset E^{0}$ be the $\mathrm{H}-\mathrm{N}$-semistable factor of $E^{0}$ with the highest slope and let $\mu:=\mu(F)$. Then $(L F)^{0} \hookrightarrow\left(L E^{0}\right)^{0}$ and so $(L F)^{0}=0$. Let $\operatorname{ch}(F)=\left(a_{0}, \mu a_{0}, a_{2}, a_{3}\right)$. Now suppose $\mu>-1 / 2$ for a contradiction. Then $L F \in \mathcal{T}_{0}$ and $F$ fits into the $\operatorname{Coh}(X)$-short exact sequence

$$
0 \rightarrow F \rightarrow E^{0} \rightarrow G \rightarrow 0
$$

for some $G \in \mathrm{HN}(-\infty, 0]$. By Proposition 4.5, the sheaf $E^{0}$ is reflexive. Since $G$ is torsion-free, it follows that $F$ is also reflexive. Apply the Fourier-Mukai transform $\Phi$ and consider the long exact sequence of $\operatorname{Coh}(X)$-cohomology. Then we have $F \in V_{\operatorname{Coh}(X)}^{\Phi}(2,3)$ and

$$
0 \rightarrow G^{1} \rightarrow F^{2} \rightarrow E^{02} \rightarrow \cdots
$$

is an exact sequence in $\operatorname{Coh}(X)$. From the convergence of the Mukai spectral sequence (4.1) for $E, E^{02} \cong E^{10}$ and $E^{10} \in H(-\infty, 0]$ by Proposition 4.7. Also, by Proposition 4.16, we have $G^{1} \in \mathrm{HN}(-\infty, 0]$. So $F^{2} \in \mathrm{HN}(-\infty, 0]$ and we have $\ell^{2} \operatorname{ch}_{1}\left(F^{2}\right) \leqslant 0$. Moreover, by Proposition 4.7, we have $F^{3} \in \mathrm{HN}(0,+\infty]$ and so $\ell^{2} \operatorname{ch}_{1}\left(F^{3}\right) \geqslant 0$. Therefore $\ell^{2} \operatorname{ch}_{1}(\Phi(F)) \leqslant 0$ and so $\operatorname{ch}(\Phi(F))=$ $\left(a_{3},-a_{2}, \mu a_{0},-a_{0}\right)$ implies

$$
a_{2} \ell^{3}=2 \ell \operatorname{ch}_{2}(F) \geqslant 0 .
$$

Apply the Fourier-Mukai transform $\Phi L$ to the short exact sequence (4.5) and consider the long exact sequence of $\operatorname{Coh}(X)$-cohomology. Then we have the $\operatorname{Coh}(X)$-long exact sequence

$$
0 \rightarrow(L G)^{0} \rightarrow(L F)^{1} \rightarrow\left(L E^{0}\right)^{1} \rightarrow \cdots .
$$

Here $\left(L E^{0}\right)^{1} \in \mathcal{F}_{0}$ and so $(L F)^{1} \in \mathcal{F}_{0}$. By Corollary 4.17, we have $(L F)^{2} \in \operatorname{HN}(0,+\infty]$. So $\ell^{2} \operatorname{ch}_{1}\left(L F^{1}\right) \leqslant 0$ and $\ell^{2} \operatorname{ch}_{1}\left(L F^{2}\right) \geqslant 0$, which imply $\ell^{2} \operatorname{ch}_{1}(\Phi(L F)) \geqslant 0$. Hence

$$
\left(a_{0}+2 \mu a_{0}+a_{2}\right) \ell^{3}=2 \ell \operatorname{ch}_{2}(L F) \leqslant 0 .
$$

Here, by assumption $2 \mu+1>0$, and we have already obtained $a_{2} \geqslant 0$. Hence $(2 \mu+1) a_{0}+a_{2}>0$, which is not possible. This is the contradiction required to complete the proof.

Proposition 4.19. Let $E \in \mathrm{HN}[-1,0]$; then $E^{3} \in \mathrm{HN}\left[\frac{1}{2},+\infty\right]$.

Proof. From the duality spectral sequence (4.2) for $E$ we have $\left(E^{*}\right)^{0} \cong(-1)^{*}\left(E^{3}\right)^{*}$. Here $E^{*} \in$ $\mathrm{HN}[0,1]$ and so by Propositions 4.6 and 4.18 , we have $\left(E^{*}\right)^{0} \in \mathrm{HN}\left(-\infty,-\frac{1}{2}\right]$. Hence $\left(E^{3}\right)^{*} \in$ $\mathrm{HN}\left(-\infty,-\frac{1}{2}\right]$ and so $E^{3} \in \mathrm{HN}\left[\frac{1}{2},+\infty\right]$, as required.

THEOREM 4.20. We have the following inclusions:

(i) $L \Phi(\mathcal{B}) \subset\langle\mathcal{B}, \mathcal{B}[-1], \mathcal{B}[-2]\rangle$;

(ii) $\Phi L^{-1}[1](\mathcal{B}) \subset\langle\mathcal{B}, \mathcal{B}[-1], \mathcal{B}[-2]\rangle$.

Proof. (i) We can visualize $\mathcal{B}$ as follows:

$$
\mathcal{B}=\langle\mathcal{F}[1], \mathcal{T}\rangle: \underbrace{A}_{-1}
$$

If $E \in \mathcal{F}=\mathrm{HN}\left(-\infty, \frac{1}{2}\right]$, then by Propositions 4.6 and 4.18 , we have $L E^{0} \in \mathcal{F}$. Also, by Proposition 4.7, we have $L E^{3} \in \mathrm{HN}(1,+\infty] \subset \mathcal{F}$. Therefore $L \Phi(E)$ has $\mathcal{B}$-cohomology in positions 1 , 


\section{A. Maciocia and D. Piyaratne}

2 and 3 ; that is,

$$
L \Phi(\mathcal{F})[1] \subset\langle\mathcal{B}, \mathcal{B}[-1], \mathcal{B}[-2]\rangle
$$

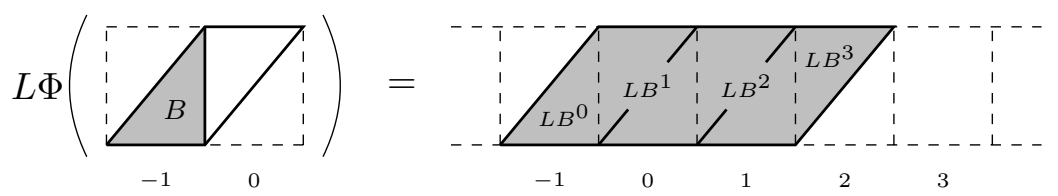

On the other hand, if $E \in \mathcal{T}=\operatorname{HN}\left(\frac{1}{2},+\infty\right]$, then by Proposition 4.6 , we have $L E^{3}=0$ and by Corollary 4.17, we have $L E^{2} \in \mathrm{HN}(1,+\infty] \subset \mathcal{T}$. So $L \Phi(E)$ has $\mathcal{B}$-cohomology in positions 0,1 and 2; that is,

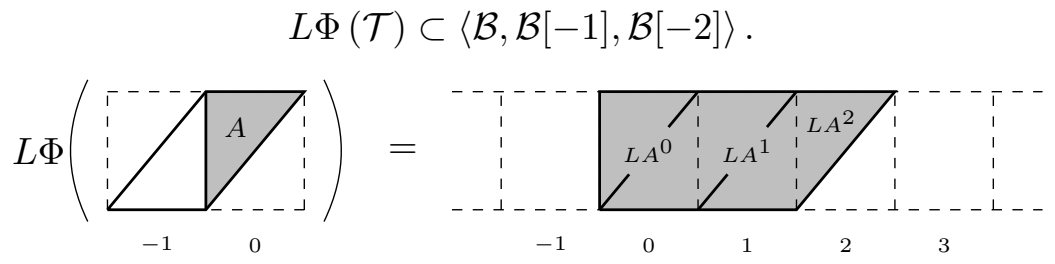

Hence $L \Phi(\mathcal{B}) \subset\langle\mathcal{B}, \mathcal{B}[-1], \mathcal{B}[-2]\rangle$, as $\mathcal{B}=\langle\mathcal{F}[1], \mathcal{T}\rangle$.

(ii) We can use Propositions 4.6, 4.7, 4.19 and 4.16 in a way similar to that used for the proof of part (i).

\section{5. (Semi)stable sheaves with the Chern character $(r, 0,0, \chi)$}

In this section we shall consider sheaves $E$ with $\operatorname{ch}_{k}(E)=0$ for $k=1,2$ which arise as the $\operatorname{Coh}(X)$ cohomology of some of the tilt-stable objects, for example when $F \in \mathcal{B}$ is a tilt-stable object with $\nu(F)=0$ and $F_{i}:=H_{\operatorname{Coh}(X)}^{i}(F)$. By Proposition 3.1, if $\mu\left(F_{-1}\right)=0$, then $\operatorname{ch}_{k}\left(F_{-1}\right)=0$, and if $\mu\left(F_{0}\right)=1$, then $\operatorname{ch}_{k}\left(L^{-1} F_{0}\right)=0$ for $k=1,2$.

We would like to show that such sheaves can only take on a very special form.

TheOREM 5.1. Let $E$ be a slope-semistable sheaf with $\operatorname{ch}_{k}(E)=0$ for $k=1,2$. Then $E^{* *}$ is a homogeneous bundle. In other words, $E^{* *}$ is filtered with quotients from $\mathbf{P i c}^{0}(X)$.

Proof. Assume the opposite for a contradiction. Then there exists a stable reflexive sheaf $E$ with $\operatorname{ch}_{k}(E)=0$ for $k=1,2$ and $H^{k}\left(X, E \otimes \mathscr{P}_{x}\right)=0$ for $k=0,3$ and any $x \in X$. By a result of Simpson ([Sim92, Theorem 2]), we have $\operatorname{ch}_{3}(E)=0$. Therefore, $\operatorname{ch}(E)=(r, 0,0,0)$ for some positive integer $r$.

Since $H^{k}\left(X, E \otimes \mathscr{P}_{x}\right)=0$ for $k=0,3$ and any $x \in X$, we have $E^{0}=E^{3}=0$. By Proposition 4.16, we have $E^{1} \in \mathrm{HN}(-\infty, 0]$ and $E^{2} \in \mathrm{HN}[0,+\infty]$. So $\ell^{2} \operatorname{ch}_{1}\left(E^{1}\right) \leqslant 0$ and $\ell^{2} \operatorname{ch}_{1}\left(E^{2}\right) \geqslant 0$. Therefore $\ell^{2} \operatorname{ch}_{1}(\Phi(E)) \geqslant 0$, which implies $\ell \operatorname{ch}_{2}(E) \leqslant 0$. Since $\operatorname{ch}_{2}(E)=0$, we obtain $\operatorname{ch}_{1}\left(E^{1}\right)=\operatorname{ch}_{1}\left(E^{2}\right)=0$. Consequently,

$$
\operatorname{ch}\left(E^{1}\right)=(a, 0,-b, c), \quad \operatorname{ch}\left(E^{2}\right)=(a, 0,-b,-r+c),
$$

for some $a>0$ and $b \geqslant 0$. Moreover, we have $E^{1} \in \mathrm{HN}[0]$.

If $E^{13} \neq 0$, then $E^{1}$ fits into a $\operatorname{Coh}(X)$-short exact sequence of the form $0 \rightarrow K_{1} \rightarrow E^{1} \rightarrow$ $\mathscr{P}_{z_{1}} \mathcal{I}_{C_{1}} \rightarrow 0$. Then $K_{1} \in \mathrm{HN}[0]$ and we have the following exact sequence in $\operatorname{Coh}(X)$ :

$$
\cdots \rightarrow K_{1}^{3} \rightarrow E^{13} \rightarrow \mathcal{O}_{-z_{1}} \rightarrow 0 \text {. }
$$




\section{FMTS AND STABILITY CONDITIONS ON ABELIAN THREEFOLDS}

If $K_{1}^{3} \neq 0$, then $K_{1}$ fits into a $\operatorname{Coh}(X)$-short exact sequence $0 \rightarrow K_{2} \rightarrow K_{1} \rightarrow \mathscr{P}_{z_{2}} \mathcal{I}_{C_{2}} \rightarrow 0$. So $K_{2} \in \mathrm{HN}[0]$ and we have the following exact sequence in $\operatorname{Coh}(X)$ :

$$
\cdots \rightarrow K_{2}^{3} \rightarrow K_{1}^{3} \rightarrow \mathcal{O}_{-z_{2}} \rightarrow 0 \text {. }
$$

We can continue this process for only a finite number of steps since $\operatorname{rk}\left(E^{1}\right)<+\infty$ and hence $E^{13}$ is filtered by skyscraper sheaves. Moreover, from the convergence of the Mukai spectral sequence (4.1) for $E$, we have the $\operatorname{Coh}(X)$-short exact sequence

$$
0 \rightarrow E^{20} \rightarrow E^{12} \rightarrow Q \rightarrow 0,
$$

where $Q$ is a subsheaf of $(-1)^{*} E$, and so $Q \in \mathrm{HN}(-\infty, 0]$. By Proposition 4.7, we have $E^{20} \in$ $\mathrm{HN}(-\infty, 0]$. This implies $E^{12} \in \mathrm{HN}(-\infty, 0]$. Then $\ell^{2} \operatorname{ch}_{1}\left(\Phi\left(E^{1}\right)\right) \leqslant 0$ and so $-b \ell^{3}=2 \ell \operatorname{ch}_{2}\left(E^{1}\right) \geqslant$ 0 . Hence $b=0$. By Proposition 4.8 , the sheaf $E^{1}$ is reflexive. Since $E^{1}$ is an element of $\mathrm{HN}[0]$, it is slope-semistable. So by [Sim92, Theorem 2], we have $c=\operatorname{ch}_{3}\left(E^{1}\right)=0$. Therefore $\operatorname{ch}\left(\Phi\left(E^{1}\right)\right)=$ $(0,0,0,-a)$. Since $E^{13}$ is an element of $\operatorname{Coh}^{0}(X)$, we have $\operatorname{ch}_{k}\left(E^{12}\right)=0$ for $k=0,1,2$. So $E^{12} \in \operatorname{HN}(0,+\infty]$. Therefore $E^{12}=0$ and we have the $\operatorname{Coh}(X)$-short exact sequence

$$
0 \rightarrow(-1)^{*} E \rightarrow E^{21} \rightarrow E^{13} \rightarrow 0 .
$$

Since $E^{13}$ is an element of $\operatorname{Coh}^{0}(X)$ and $E$ is locally free, $\operatorname{Ext}^{1}\left(E^{13},(-1)^{*} E\right)=0$. Hence $E^{21} \cong$ $(-1)^{*} E \oplus E^{13}$. Since $E^{21}$ is an element of $V_{\mathrm{Coh}(X)}^{\Phi}(2)$, we have $E^{13}=0$ and so $E \in V_{\mathrm{Coh}(X)}^{\Phi}(2)$. Therefore $\operatorname{ch}\left(E^{2}\right)=(0,0,0,-r)$. But it is not possible to have $-r>0$. This is the contradiction required to complete the proof.

Remark 5.2. Theorem 5.1 can be interpreted as saying that if a vector bundle $E$ over $X$ satisfies $c_{1}(E)=0=c_{2}(E)$, then it cannot carry a non-flat Hermitian-Einstein connection. This is analogous to the case where there are no charge one $\mathrm{SU}(r)$-instantons on an abelian surface. This is proved in a slick way using the Fourier-Mukai transform and it would be good to avoid the direct proof given for Theorem 5.1 as it would follow more directly from Theorem 6.10.

\section{Auto-equivalences of $\mathcal{A}_{\sqrt{3} \ell / 2, \ell / 2}$ under Fourier-Mukai transforms}

Let us define the Fourier-Mukai transforms $\Psi=L \Phi$ and $\hat{\Psi}=\Phi L^{-1}[1]$. Then by Theorem 4.20, the images of an object from $\mathcal{B}$ under $\Psi$ and $\hat{\Psi}$ are complexes whose $\mathcal{B}$-cohomology can only be non-zero in positions 0,1 or 2 . We have $\Psi \circ \hat{\Psi} \cong(-1)^{*} \operatorname{id}_{D^{b}(X)}[-2]$ and $\hat{\Psi} \circ \Psi \cong(-1)^{*} \operatorname{id}_{D^{b}(X)}[-2]$. This gives us the following convergence of spectral sequences.

Proposition 6.1. We have

$$
\begin{aligned}
& E_{2}^{p, q}=\Psi_{\mathcal{B}}^{p} \hat{\Psi}_{\mathcal{B}}^{q}(E) \Longrightarrow H_{\mathcal{B}}^{p+q-2}\left((-1)^{*} E\right), \\
& E_{2}^{p, q}=\hat{\Psi}_{\mathcal{B}}^{p} \Psi_{\mathcal{B}}^{q}(E) \Longrightarrow H_{\mathcal{B}}^{p+q-2}\left((-1)^{*} E\right)
\end{aligned}
$$

for $E \in \mathcal{B}$, where $\Psi_{\mathcal{B}}^{i}(F):=H_{\mathcal{B}}^{i}(\Psi(F))$ and $\hat{\Psi}_{\mathcal{B}}^{i}(F):=H_{\mathcal{B}}^{i}(\hat{\Psi}(F))$.

This convergence of the spectral sequences for $E \in \mathcal{B}$ looks similar to the convergence of certain spectral sequences in an abelian surface for coherent sheaves. See [BBR09, Mac96, Yos09] for further details.

Recall that if $B_{1}, B_{2} \in \mathcal{B}$, then $\operatorname{Ext}^{i}\left(B_{1}, B_{2}\right)=0$ for any $i<0$.

Proposition 6.2. For $E \in D^{b}(X)$ we have

$$
\Im(Z(\Psi(E)))=-\Im(Z(E)) \quad \text { and } \quad \Im(Z(\hat{\Psi}(E)))=-\Im(Z(E)) \text {. }
$$




\section{A. Maciocia and D. Piyaratne}

Proof. Let $\operatorname{ch}(E)=\left(a_{0}, a_{1}, a_{2}, a_{3}\right)$. Then $\Im(Z(E))=\frac{3}{4} \sqrt{3}\left(a_{2}-a_{1}\right)$. Also, we have $\operatorname{ch}(\Psi(E))=$ $\left(*, a_{3}-a_{2}, a_{3}-2 a_{2}+a_{1}, *\right)$ and $\operatorname{ch}(\hat{\Psi}(E))=\left(*, a_{2}-2 a_{1}+a_{0},-a_{1}+a_{0}, *\right)$. Then $\Im(Z(\Psi(E)))=$ $\Im(Z(\hat{\Psi}(E)))=-\frac{3}{4} \sqrt{3}\left(a_{2}-a_{1}\right)$, as required.

From Propositions 2.7 and 3.1 and Theorem 5.1, we obtain the following result.

Remark 6.3. Let $E \in \mathcal{B}$. If $E \in \operatorname{HN}^{\nu}(-\infty, 0)$, then $\mu^{+}\left(E_{-1}\right)<0$. If on the other hand, $E \in$ $\operatorname{HN}^{\nu}(0,+\infty]$, then $\mu^{-}\left(E_{0}\right)>1$.

For tilt-stable $E$ with $\nu(E)=0$, we have $\mu^{+}\left(E_{-1}\right) \leqslant 0$ and $\mu^{-}\left(E_{0}\right) \geqslant 1$. Moreover, if $\mu\left(E_{-1}\right)=0$, then $E_{-1}=\mathscr{P}_{x}$ for some $x \in X$; and if $\mu\left(E_{0}\right)=1$, then $E_{0}^{* *}=L \mathscr{P}_{x}$ for some $x \in X$.

Proposition 6.4. Let $E \in \mathcal{T}^{\prime}$.

(i) $H_{\operatorname{Coh}(X)}^{0}\left(\hat{\Psi}_{\mathcal{B}}^{2}(E)\right)=0$.

(ii) If $\hat{\Psi}_{\mathcal{B}}^{2}(E) \neq 0$, then $\Im\left(Z\left(\hat{\Psi}_{\mathcal{B}}^{2}(E)\right)\right)>0$.

Proof. (i) For any $x \in X$, we have

$$
\begin{aligned}
\operatorname{Hom}\left(\hat{\Psi}_{\mathcal{B}}^{2}(E), \mathcal{O}_{x}\right) & \cong \operatorname{Hom}\left(\hat{\Psi}_{\mathcal{B}}^{2}(E), \hat{\Psi}_{\mathcal{B}}^{2}\left(L \mathscr{P}_{-x}\right)\right) \\
& \cong \operatorname{Hom}\left(\hat{\Psi}(E), \hat{\Psi}\left(L \mathscr{P}_{-x}\right)\right) \\
& \cong \operatorname{Hom}\left(E, L \mathscr{P}_{-x}\right)=0
\end{aligned}
$$

since $E \in \mathcal{T}^{\prime}$ and $L \mathscr{P}_{-x} \in \mathcal{F}^{\prime}$. Therefore $H_{\mathrm{Coh}(X)}^{0}\left(\hat{\Psi}_{\mathcal{B}}^{2}(E)\right)=0$, as required.

(ii) From part (i), we have $\hat{\Psi}_{\mathcal{B}}^{2}(E) \cong A[1]$ for some non-zero $A \in \operatorname{HN}(-\infty, 1 / 2]$.

Consider the convergence of the spectral sequence

$$
E_{2}^{p, q}=\hat{\Psi}_{\operatorname{Coh}(X)}^{p}\left(H_{\operatorname{Coh}(X)}^{q}(E)\right) \Longrightarrow \hat{\Psi}_{\operatorname{Coh}(X)}^{p+q}(E)
$$

[] for $E$. Let $E_{i}:=H_{\operatorname{Coh}(X)}^{i}(E)$. Then by Remark 6.3, we have $E_{0} \in \mathrm{HN}(1,+\infty]$ and so by Corollary 4.17 and Proposition 4.7, we have

$$
\left(L^{-1} E_{0}\right)^{2},\left(L^{-1} E_{-1}\right)^{3} \in \mathrm{HN}(0,+\infty] .
$$

Therefore, from the convergence of the spectral sequence for $E$ given above, we have

$$
A \in \mathrm{HN}\left(-\infty, \frac{1}{2}\right] \cap \mathrm{HN}(0,+\infty]=\mathrm{HN}\left(0, \frac{1}{2}\right] .
$$

Let $\operatorname{ch}(A)=\left(a_{0}, a_{1}, a_{2}, a_{3}\right)$. Then from the B-G inequalities for all the H-N-semistable factors of $A$, we have

$$
\Im\left(Z\left(\hat{\Psi}_{\mathcal{B}}^{2}(E)\right)\right)=\Im(Z(A[1]))=\frac{3}{4} \sqrt{3}\left(a_{1}-a_{2}\right)>0,
$$

as required.

Proposition 6.5. Let $E \in \mathcal{F}^{\prime}$.

(i) $H_{\operatorname{Coh}(X)}^{-1}\left(\hat{\Psi}_{\mathcal{B}}^{0}(E)\right)=0$.

(ii) If $\hat{\Psi}_{\mathcal{B}}^{0}(E) \neq 0$, then $\Im\left(Z\left(\hat{\Psi}_{\mathcal{B}}^{0}(E)\right)\right)<0$. 


\section{FMTS AND STABILITY CONDITIONS ON ABELIAN THREEFOLDS}

Proof. (i) Let $x \in X$. Then we have

$$
\begin{aligned}
\operatorname{Hom}\left(\hat{\Psi}_{\mathcal{B}}^{0}(E), \mathcal{O}_{x}[1]\right) & \cong \operatorname{Hom}\left(\Psi \hat{\Psi}^{0}(E), \Psi\left(\mathcal{O}_{x}[1]\right)\right) \\
& \cong \operatorname{Hom}\left(\Psi_{\mathcal{B}}^{2} \hat{\Psi}_{\mathcal{B}}^{0}(E)[-2], L \mathscr{P}_{x}[1]\right) \\
& \cong \operatorname{Hom}\left(\Psi_{\mathcal{B}}^{2} \hat{\Psi}_{\mathcal{B}}^{0}(E), L \mathscr{P}_{x}[3]\right) \\
& \cong \operatorname{Hom}\left(L \mathscr{P}_{x}, \Psi_{\mathcal{B}}^{2} \hat{\Psi}_{\mathcal{B}}^{0}(E)\right)^{*}
\end{aligned}
$$

From the convergence of the spectral sequence (6.1) for $E$, we have the $\mathcal{B}$-short exact sequence

$$
0 \rightarrow \Psi_{\mathcal{B}}^{0} \hat{\Psi}_{\mathcal{B}}^{1}(E) \rightarrow \Psi_{\mathcal{B}}^{2} \hat{\Psi}_{\mathcal{B}}^{0}(E) \rightarrow F \rightarrow 0,
$$

where $F$ is a subobject of $(-1)^{*} E$ and so $F \in \mathcal{F}^{\prime}$. Moreover, by the H-N filtration, $F$ fits into the following $\mathcal{B}$-short exact sequence:

$$
0 \rightarrow F_{0} \rightarrow F \rightarrow F_{1} \rightarrow 0
$$

where $F_{0} \in \mathrm{HN}^{\nu}[0]$ and $F_{1} \in \mathrm{HN}^{\nu}(-\infty, 0)$. Since $L \mathscr{P}_{x}$ is an element of $\mathrm{HN}^{\nu}[0]$, we have

$$
\operatorname{Hom}\left(L \mathscr{P}_{x}, F_{1}\right)=0 \text {. }
$$

Moreover, $F_{0}$ has a filtration of $\nu$-stable objects $F_{0, i}$ with $\nu\left(F_{0, i}\right)=0$. By Proposition 2.9, each $F_{0, i}$ fits into a non-splitting $\mathcal{B}$-short exact sequence

$$
0 \rightarrow F_{0, i} \rightarrow M_{i} \rightarrow T_{i} \rightarrow 0
$$

for some $T_{i} \in \operatorname{Coh}^{0}(X)$ such that $M_{i}[1] \in \mathcal{A}$ is a minimal object. Moreover, $L \mathscr{P}_{x}[1] \in \mathcal{A}$ is a minimal object. Consequently, for finitely many $x \in X$, we can have $L \mathscr{P}_{x} \cong M_{i}$ for some $i$. So for a generic $x \in X$, we have $\operatorname{Hom}\left(L \mathscr{P}_{x}, M_{i}\right)=0$ and therefore $\operatorname{Hom}\left(L \mathscr{P}_{x}, F_{0, i}\right)=0$, which implies $\operatorname{Hom}\left(L \mathscr{P}_{x}, F_{0}\right)=0$. Hence for a generic $x \in X$, we have $\operatorname{Hom}\left(L \mathscr{P}_{x}, F\right)=0$.

On the other hand, we have

$$
\begin{aligned}
\operatorname{Hom}\left(L \mathscr{P}_{x}, \Psi_{\mathcal{B}}^{0} \hat{\Psi}_{\mathcal{B}}^{1}(E)\right) & \cong \operatorname{Hom}\left(\Psi_{\mathcal{B}}^{0}\left(\mathcal{O}_{x}\right), \Psi_{\mathcal{B}}^{0} \hat{\Psi}_{\mathcal{B}}^{1}(E)\right) \\
& \cong \operatorname{Hom}\left(\Psi\left(\mathcal{O}_{x}\right), \Psi \hat{\Psi}_{\mathcal{B}}^{1}(E)\right) \\
& \cong \operatorname{Hom}\left(\mathcal{O}_{x}, \hat{\Psi}_{\mathcal{B}}^{1}(E)\right)
\end{aligned}
$$

Here $\hat{\Psi}_{\mathcal{B}}^{1}(E)$ fits into the $\mathcal{B}$-short exact sequence

$$
0 \rightarrow H_{\mathrm{Coh}(X)}^{-1}\left(\hat{\Psi}_{\mathcal{B}}^{1}(E)\right)[1] \rightarrow \hat{\Psi}_{\mathcal{B}}^{1}(E) \rightarrow H_{\mathrm{Coh}(X)}^{0}\left(\hat{\Psi}_{\mathcal{B}}^{1}(E)\right) \rightarrow 0,
$$

where $H_{\mathrm{Coh}(X)}^{-1}\left(\hat{\Psi}_{\mathcal{B}}^{1}(E)\right)$ is torsion free and $H_{\mathrm{Coh}(X)}^{0}\left(\hat{\Psi}_{\mathcal{B}}^{1}(E)\right)$ can have torsion supported on a zero-scheme of finite length. Hence for generic $x \in X$, we have $\operatorname{Hom}\left(\mathcal{O}_{x}, \hat{\Psi}_{\mathcal{B}}^{1}(E)\right)=0$. Consequently for generic $x \in X$, the equalities $\operatorname{Hom}\left(L \mathscr{P}_{x}, \Psi_{\mathcal{B}}^{0} \hat{\Psi}_{\mathcal{B}}^{1}(E)\right)=\operatorname{Hom}\left(L \mathscr{P}_{x}, F\right)=0$ imply $\operatorname{Hom}\left(L \mathscr{P}_{x}, \Psi_{\mathcal{B}}^{2} \hat{\Psi}_{\mathcal{B}}^{0}(E)\right)=0$. Therefore $\operatorname{Hom}\left(\hat{\Psi}_{\mathcal{B}}^{0}(E), \mathcal{O}_{x}[1]\right)=0$ for generic $x \in X$. But $H_{\mathrm{Coh}(X)}^{-1}\left(\hat{\Psi}_{\mathcal{B}}^{0}(E)\right)$ is torsion free and so $H_{\mathrm{Coh}(X)}^{-1}\left(\hat{\Psi}_{\mathcal{B}}^{0}(E)\right)=0$, as required.

(ii) From part (i), we have $\hat{\Psi}_{\mathcal{B}}^{0}(E) \cong A$ for some coherent non-zero sheaf $A \in \operatorname{HN}\left(\frac{1}{2},+\infty\right]$. For any $x \in X$, we have

$$
\begin{aligned}
\operatorname{Ext}^{1}\left(\mathcal{O}_{x}, A\right) & \cong \operatorname{Ext}^{1}\left(\mathcal{O}_{x}, \hat{\Psi}_{\mathcal{B}}^{0}(E)\right) \cong \operatorname{Hom}\left(\Psi\left(\mathcal{O}_{x}\right), \Psi \hat{\Psi}_{\mathcal{B}}^{0}(E)[1]\right) \\
& \cong \operatorname{Hom}\left(L \mathscr{P}_{x}, \Psi^{2} \hat{\Psi}_{\mathcal{B}}^{0}(E)[-1]\right)=0 .
\end{aligned}
$$

So $A \in \operatorname{Coh}^{\geqslant 2}(X)$, and if $\operatorname{ch}(A)=\left(a_{0}, a_{1}, a_{2}, a_{3}\right)$, then $a_{1}>0$. 


\section{A. Maciocia and D. Piyaratne}

Apply the Fourier-Mukai transform $\Psi$ to $\hat{\Psi}_{\mathcal{B}}^{0}(E)$. Since $\hat{\Psi}_{\mathcal{B}}^{0}(E)$ is an element of $V_{\mathcal{B}}^{\Psi}(2)$, the transform $\Psi_{\mathcal{B}}^{2} \hat{\Psi}_{\mathcal{B}}^{0}(E) \in \mathcal{B}$ has $\operatorname{Coh}(X)$-cohomology $L A^{1}$ in position -1 and $L A^{2}$ in position 0 . So we have $A \in V_{\mathrm{Coh}(X)}^{\Phi}(1,2), L A^{1} \in \mathrm{HN}\left(-\infty, \frac{1}{2}\right]$ and, by Corollary $4.17, A^{2} \in \operatorname{HN}(0,+\infty]$. Therefore $\ell^{2} \operatorname{ch}_{1}\left(A^{1}\right) \leqslant 0$ and $\ell^{2} \operatorname{ch}_{1}\left(A^{2}\right) \geqslant 0$. Hence

$$
a_{2} \ell^{3}=2 \ell \operatorname{ch}_{2}(A)=-\ell^{2} \operatorname{ch}_{1}(\Phi(A))=-\ell^{2} \operatorname{ch}_{1}\left(A^{2}\right)+\ell^{2} \operatorname{ch}_{1}\left(A^{1}\right) \leqslant 0 .
$$

So we have

$$
\Im\left(Z\left(\hat{\Psi}_{\mathcal{B}}^{0}(E)\right)\right)=\Im(Z(A))=\frac{3}{4} \sqrt{3}\left(a_{2}-a_{1}\right)<0,
$$

as required.

Proposition 6.6. (i) Let $E \in \mathcal{T}^{\prime}$. We have $H_{\mathrm{Coh}(X)}^{0}\left(\Psi_{\mathcal{B}}^{2}(E)\right)=0$, and if $\Psi_{\mathcal{B}}^{2}(E) \neq 0$, then $\Im\left(Z\left(\Psi_{\mathcal{B}}^{2}(E)\right)\right)>0$.

(ii) Let $E \in \mathcal{F}^{\prime}$. We have $H_{\operatorname{Coh}(X)}^{-1}\left(\Psi_{\mathcal{B}}^{0}(E)\right)=0$, and if $\Psi_{\mathcal{B}}^{0}(E) \neq 0$, then $\Im Z\left(\Psi_{\mathcal{B}}^{0}(E)\right)<0$.

Proof. (i) Let $E \in \mathcal{T}^{\prime}$. The proof of the first assertion is similar to the proof of part (i) of Proposition 6.4. From the first assertion, we have $\Psi_{\mathcal{B}}^{2}(E) \cong A[1]$ for some coherent non-zero sheaf $A \in \mathrm{HN}\left(-\infty, \frac{1}{2}\right]$. Let $\operatorname{ch}(A)=\left(a_{0}, a_{1}, a_{2}, a_{3}\right)$. Then $\operatorname{ch}\left(L^{-1} A\right)=\left(a_{0}, a_{1}-a_{0}, a_{2}-2 a_{1}+a_{0}, *\right)$ and so $a_{1}-a_{0}<0$.

Apply the Fourier-Mukai transform $\hat{\Psi}$ to $\Psi_{\mathcal{B}}^{2}(E)$. Since $\Psi_{\mathcal{B}}^{2}(E)$ is an element of $V_{\mathcal{B}}^{\hat{\Psi}}(0)$, the transform $\hat{\Psi}_{\mathcal{B}}^{0} \Psi_{\mathcal{B}}^{2}(E) \in \mathcal{B}$ has $\operatorname{Coh}(X)$-cohomology $\left(L^{-1} A\right)^{1}$ in position -1 and $\left(L^{-1} A\right)^{2}$ in position 0 . So we have $\left(L^{-1} A\right)^{2} \in \operatorname{HN}\left(\frac{1}{2},+\infty\right]$, and, by Proposition 4.16, $\left(L^{-1} A\right)^{1} \in \mathrm{HN}(-\infty, 0]$. Therefore $\ell^{2} \operatorname{ch}_{1}\left(\left(L^{-1} A\right)^{1}\right) \leqslant 0$ and $\ell^{2} \operatorname{ch}_{1}\left(\left(L^{-1} A\right)^{2}\right) \geqslant 0$. Hence we have

$$
\begin{aligned}
\left(a_{2}-2 a_{1}+a_{0}\right) \ell^{3} & =2 \ell \operatorname{ch}_{2}\left(L^{-1} A\right)=-\ell^{2} \operatorname{ch}_{1}\left(\Phi\left(L^{-1} A\right)\right) \\
& =-\ell^{2} \operatorname{ch}_{1}\left(\left(L^{-1} A\right)^{2}\right)+\ell^{2} \operatorname{ch}_{1}\left(\left(L^{-1} A\right)^{1}\right) \leqslant 0,
\end{aligned}
$$

and consequently

$$
\begin{aligned}
\Im\left(Z\left(\Psi_{\mathcal{B}}^{2}(E)\right)\right) & =\Im(Z(A[1]))=\frac{3}{4} \sqrt{3}\left(a_{1}-a_{2}\right) \\
& =-\frac{3}{4} \sqrt{3}\left(\left(a_{1}-a_{0}\right)+\left(a_{2}-2 a_{1}+a_{0}\right)\right)>0,
\end{aligned}
$$

as required.

(ii) Let $E \in \mathcal{F}^{\prime}$. The proof of the first assertion is similar to the proof of part (i) of Proposition 6.5. From the first assertion, we have $\Psi_{\mathcal{B}}^{0}(E) \cong A$ for some non-zero $A \in \operatorname{HN}\left(\frac{1}{2},+\infty\right]$.

Consider the convergence of the spectral sequence

$$
E_{2}^{p, q}=\Psi_{\mathrm{Coh}(X)}^{p}\left(H_{\mathrm{Coh}(X)}^{q}(E)\right) \Longrightarrow \Psi_{\mathrm{Coh}(X)}^{p+q}(E)
$$

for $E$. Let $E_{i}:=H_{\operatorname{Coh}(X)}^{i}(E)$. Then by Remark 6.3 , we have $E_{-1} \in \mathrm{HN}(-\infty, 0]$ and so by Propositions 4.16 and 4.7 , we have

$$
L E_{-1}^{1} \in \mathrm{HN}(-\infty, 1] \quad \text { and } \quad L E_{0}^{0} \in \mathrm{HN}(-\infty, 1] .
$$

Therefore, from the convergence of the spectral sequence for $E$ given above, we have

$$
A \in \mathrm{HN}\left(\frac{1}{2},+\infty\right] \cap \mathrm{HN}(-\infty, 1]=\mathrm{HN}\left(\frac{1}{2}, 1\right] .
$$

Also, $A$ is reflexive, as $L E_{0}^{0}$ and $L E_{-1}^{1}$ are reflexive sheaves by Propositions 4.5 and 4.8 . Let $\operatorname{ch}(A)=\left(a_{0}, a_{1}, a_{2}, a_{3}\right)$. Then from the B-G inequalities for all the H-N-semistable factors of $A$, we have

$$
\Im\left(Z\left(\Psi_{\mathcal{B}}^{0}(E)\right)\right)=\Im(Z(A))=\frac{3}{4} \sqrt{3}\left(a_{2}-a_{1}\right) \leqslant 0 .
$$




\section{FMTS AND STABILITY CONDITIONS ON ABELIAN THREEFOLDS}

Equality holds when $A \in \mathrm{HN}[1]$ with $\operatorname{ch}(A)=\left(a_{0}, a_{0}, a_{0}, *\right)$. Then, by considering a JordanHölder filtration for $A$ together with Theorem 5.1, we see that $L^{-1} A$ has a filtration of ideal sheaves $\mathscr{P}_{x_{i}} \mathcal{I}_{Z_{i}}$ of some zero-subschemes. Here $\Psi_{\mathcal{B}}^{0}(E) \cong A \in V_{\mathcal{B}}^{\hat{\Psi}}(2)$ implies that we have $L^{-1} A \in V_{\mathrm{Coh}(X)}^{\Phi}(2,3)$. An easy induction on the rank of $A$ also shows that $L^{-1} A \in V_{\mathrm{Coh}(X)}^{\Phi}(1,3)$ and so $L^{-1} A \in V_{\mathrm{Coh}(X)}^{\Phi}(3)$. But then $Z_{i}=\emptyset$ for all $i$. Therefore $\hat{\Psi}_{\mathcal{B}}^{2} \Psi_{\mathcal{B}}^{0}(E) \in \operatorname{Coh}^{0}(X)$. Now consider the convergence of the spectral sequence (6.1) for $E$. We have a $\mathcal{B}$-short exact sequence

$$
0 \rightarrow \hat{\Psi}_{\mathcal{B}}^{0} \Psi_{\mathcal{B}}^{1}(E) \rightarrow \hat{\Psi}_{\mathcal{B}}^{2} \Psi_{\mathcal{B}}^{0}(E) \rightarrow F \rightarrow 0
$$

where $F$ is a subobject of $(-1)^{*} E$, and so $F \in \mathcal{F}^{\prime}$. Then $\hat{\Psi}_{\mathcal{B}}^{2} \Psi_{\mathcal{B}}^{0}(E) \in \operatorname{Coh}^{0}(X) \subset \mathcal{T}^{\prime}$, which implies $F=0$ and $\hat{\Psi}_{\mathcal{B}}^{0} \Psi_{\mathcal{B}}^{1}(E) \cong \hat{\Psi}_{\mathcal{B}}^{2} \Psi_{\mathcal{B}}^{0}(E)$. But then we have $\Psi_{\mathcal{B}}^{0}(E) \cong(-1)^{*} \Psi_{\mathcal{B}}^{0} \hat{\Psi}_{\mathcal{B}}^{0} \Psi_{\mathcal{B}}^{1}(E)=0$. This is not possible as $\Psi_{\mathcal{B}}^{0}(E) \neq 0$. Therefore we have the strict inequality $\Im\left(Z\left(\Psi_{\mathcal{B}}^{0}(E)\right)\right)<0$, as required to complete the proof.

Lemma 6.7. (i) Let $E \in \mathcal{T}^{\prime}$; then $\hat{\Psi}_{\mathcal{B}}^{2}(E)=0$ and $\Psi_{\mathcal{B}}^{2}(E)=0$.

(ii) Let $E \in \mathcal{F}^{\prime}$; then $\hat{\Psi}_{\mathcal{B}}^{0}(E)=0$ and $\Psi_{\mathcal{B}}^{0}(E)=0$.

Proof. (i) Let $E \in \mathcal{T}^{\prime}$. From the convergence of the spectral sequence (6.1) for $E$, we have the $\mathcal{B}$-short exact sequence

$$
0 \rightarrow Q \rightarrow \Psi_{\mathcal{B}}^{0} \hat{\Psi}_{\mathcal{B}}^{2}(E) \rightarrow \Psi_{\mathcal{B}}^{2} \hat{\Psi}_{\mathcal{B}}^{1}(E) \rightarrow 0
$$

Here $Q$ is a quotient of $(-1)^{*} E \in \mathcal{T}^{\prime}$ and so $Q \in \mathcal{T}^{\prime}$. Then $\Psi_{\mathcal{B}}^{0} \hat{\Psi}_{\mathcal{B}}^{2}(E)$ fits into the $\mathcal{B}$-short exact sequence

$$
0 \rightarrow T \rightarrow \Psi_{\mathcal{B}}^{0} \hat{\Psi}_{\mathcal{B}}^{2}(E) \rightarrow F \rightarrow 0
$$

for some $T \in \mathcal{T}^{\prime}$ and $F \in \mathcal{F}^{\prime}$. Now apply the Fourier-Mukai transform $\hat{\Psi}$ and consider the long exact sequence of $\mathcal{B}$-cohomology. Then we have $\hat{\Psi}_{\mathcal{B}}^{0}(T)=0$ and $\hat{\Psi}_{\mathcal{B}}^{1}(T) \cong \hat{\Psi}_{\mathcal{B}}^{0}(F)$. By Proposition 6.5 , we have $\Im\left(Z\left(\hat{\Psi}_{\mathcal{B}}^{0}(F)\right)\right) \leqslant 0$ and by Proposition 6.4 , we have $\Im\left(Z\left(\hat{\Psi}_{\mathcal{B}}^{2}(T)\right)\right) \geqslant 0$. So $\Im(Z(\hat{\Psi}(T))) \geqslant 0$ and by Proposition 6.2 , we have $\Im(Z(T)) \leqslant 0$. Since $T$ is an element of $\mathcal{T}^{\prime}$, we have $\Im(Z(T))=0$ and $\omega^{2} \operatorname{ch}_{1}^{B}(T)=0$. Then by Lemma 1.1 , we have $T \cong T_{0}$ for some $T_{0} \in \operatorname{Coh}^{0}(X)$. But $\operatorname{Coh}^{0}(X) \subset V_{\mathcal{B}}^{\hat{\Psi}}(0)$; hence $T=0$ and therefore $Q=0$. Then $\Psi_{\mathcal{B}}^{0} \hat{\Psi}_{\mathcal{B}}^{2}(E) \cong$ $\Psi_{\mathcal{B}}^{2} \hat{\Psi}_{\mathcal{B}}^{1}(E)$ and so we have $\hat{\Psi}_{\mathcal{B}}^{2}(E) \cong(-1)^{*} \hat{\Psi}_{\mathcal{B}}^{2} \Psi_{\mathcal{B}}^{2} \hat{\Psi}_{\mathcal{B}}^{1}(E)=0$, giving the first assertion. The proof of the second assertion is similar to that of the first.

(ii) The proof of part (ii) is similar to that of part (i).

Corollary 6.8. Let $E \in \mathcal{B}$. Then $\Psi_{\mathcal{B}}^{2}(E)$ and $\hat{\Psi}_{\mathcal{B}}^{2}(E)$ are elements of $\mathcal{T}^{\prime}$ and $\Psi_{\mathcal{B}}^{0}(E)$ and $\hat{\Psi}_{\mathcal{B}}^{0}(E)$ are elements of $\mathcal{F}^{\prime}$.

Proof. By the definition of $\mathcal{T}^{\prime}$ and $\mathcal{F}^{\prime}$, the object $\Psi_{\mathcal{B}}^{2}(E)$ fits into a $\mathcal{B}$-short exact sequence

$$
0 \rightarrow T \rightarrow \Psi_{\mathcal{B}}^{2}(E) \rightarrow F \rightarrow 0
$$

for some $T \in \mathcal{T}^{\prime}$ and $F \in \mathcal{F}^{\prime}$. Now apply the Fourier-Mukai transform $\hat{\Psi}$ and consider the long exact sequence of $\mathcal{B}$-cohomology. Then by Lemma 6.7, we have $F=0$, giving $\Psi_{\mathcal{B}}^{2}(E) \in \mathcal{T}^{\prime}$.

The other results can be shown in a similar manner.

Proposition 6.9. (i) Let $E \in \mathcal{F}^{\prime}$; then $\hat{\Psi}_{\mathcal{B}}^{1}(E) \in \mathcal{F}^{\prime}$ and $\Psi_{\mathcal{B}}^{1}(E) \in \mathcal{F}^{\prime}$.

(ii) Let $E \in \mathcal{T}^{\prime}$; then $\hat{\Psi}_{\mathcal{B}}^{1}(E) \in \mathcal{T}^{\prime}$ and $\Psi_{\mathcal{B}}^{1}(E) \in \mathcal{T}^{\prime}$.

Proof. (i) By torsion theory $\hat{\Psi}_{\mathcal{B}}^{1}(E)$ fits into a $\mathcal{B}$-short exact sequence

$$
0 \rightarrow T \rightarrow \hat{\Psi}_{\mathcal{B}}^{1}(E) \rightarrow F \rightarrow 0
$$




\section{A. Maciocia and D. Piyaratne}

for some $T \in \mathcal{T}^{\prime}$ and $F \in \mathcal{F}^{\prime}$. For the first assertion, we now need to show $T=0$. Apply the Fourier-Mukai transform $\Psi$ and consider the long exact sequence of $\mathcal{B}$-cohomology. We get $\Psi_{\mathcal{B}}^{1}(T) \hookrightarrow \Psi_{\mathcal{B}}^{1} \hat{\Psi}_{\mathcal{B}}^{1}(E)$ and $T \in V_{\mathcal{B}}^{\Psi}(1)$. Also, by the convergence of the spectral sequence (6.1) for $E$, we see that $\Psi_{\mathcal{B}}^{1} \hat{\Psi}_{\mathcal{B}}^{1}(E)$ is a subobject of $(-1)^{*} E$. Hence $\Psi_{\mathcal{B}}^{1}(T) \in \mathcal{F}^{\prime}$ implies $\Im\left(Z\left(\Psi_{\mathcal{B}}^{1}(T)\right)\right) \leqslant 0$. On the other hand, by Proposition 6.2, we have $\Im\left(Z\left(\Psi_{\mathcal{B}}^{1}(T)\right)\right)=\Im(Z(T)) \geqslant 0$, as $T \in \mathcal{T}^{\prime}$. Hence $\Im(Z(T))=0$ and $T \in \mathcal{T}^{\prime}$ implies $\omega^{2} \operatorname{ch}_{1}^{B}(T)=0$. So by Lemma 1.1 , we have $T \cong T_{0}$ for some $T_{0} \in \operatorname{Coh}^{0}(X)$. Since any object of $\operatorname{Coh}^{0}(X)$ belongs to $V_{\mathcal{B}}^{\Psi}(0)$, we have $\Psi_{\mathcal{B}}^{1}(T)=0$. So $T=0$, as required to prove the first assertion. The proof of the second assertion is similar to that of the first one.

(ii) The proof of part (ii) is similar to that of part (i).

By Lemma 6.7, Corollary 6.8 and Proposition 6.9, it follows that

$$
\Psi[1]\left(\mathcal{F}^{\prime}[1]\right) \subset \mathcal{A} \quad \text { and } \quad \Psi[1]\left(\mathcal{T}^{\prime}\right) \subset \mathcal{A} .
$$

Since $\mathcal{A}=\left\langle\mathcal{F}^{\prime}[1], \mathcal{T}^{\prime}\right\rangle$, we have $\Psi[1](\mathcal{A}) \subset \mathcal{A}$. Similarly, we have $\hat{\Psi}[1](\mathcal{A}) \subset \mathcal{A}$. The isomorphisms $\hat{\Psi}[1] \circ \Psi[1] \cong(-1)^{*} \operatorname{id}_{D^{b}(X)}$ and $\Psi[1] \circ \hat{\Psi}[1] \cong(-1)^{*} \operatorname{id}_{D^{b}(X)}$ give us the following result.

Theorem 6.10. The Fourier-Mukai transforms $\Psi[1]$ and $\hat{\Psi}[1]$ give the auto-equivalences

$$
\Psi[1](\mathcal{A}) \cong \mathcal{A} \quad \text { and } \quad \hat{\Psi}[1](\mathcal{A}) \cong \mathcal{A}
$$

of the abelian category $\mathcal{A}$.

\section{ACKNOWLEDGEMENTS}

The authors would like to thank Arend Bayer and Tom Bridgeland for very useful discussions and comments. We are also grateful to Jason Lo and Yukinobu Toda for pointing out several errors. Special thanks go to the referee for a thorough reading of the paper and insightful comments that led to a substantial improvement of this paper, especially in Sections 4 and 5.

\section{REFERENCES}

AB13 D. Arcara and A. Bertram, Bridgeland-stable moduli spaces for K-trivial surfaces, With an appendix by Max Lieblich, J. Eur. Math. Soc. 15 (2013), no. 1, 1-38. http://dx.doi.org/ 10.4171/JEMS/354

BBMT14 A. Bayer, A. Bertram, E. Macrì and Y. Toda, Bridgeland Stability conditions on threefolds II: An application to Fujita's conjecture, J. Alg. Geom. 23 (2014), no. 4, 693-710. http: //dx.doi.org/10.1090/S1056-3911-2014-00637-8

BBR09 C. Bartocci, U. Bruzzo and D. Hernández Ruipérez, Fourier-Mukai and Nahm transforms in geometry and mathematical physics, Progr. Math. 276, Birkhäuser Boston, Boston, MA, 2009. http://dx.doi.org/10.1007/b11801

BMT13 A. Bayer, E. Macrì and Y. Toda, Bridgeland stability conditions on threefolds I: BogomolovGieseker type inequalities, J. Alg. Geom. 23 (2013), 117-163. http://dx.doi.org/10.1090/ S1056-3911-2013-00617-7

Bri07 T. Bridgeland, Stability conditions on triangulated categories, Ann. of Math. 166 (2007), no. 2, 317-345. http://dx.doi.org/10.4007/annals.2007.166.317

Bri08 T. Bridgeland, Stability conditions on K3 surfaces, Duke Math. J. 141 (2008), no. 2, 241-291. http://dx.doi.org/10.1215/S0012-7094-08-14122-5 


\section{FMTS AND STABILITY CONDITIONS ON ABELIAN THREEFOLDS}

Huy06 D. Huybrechts, Fourier-Mukai transforms in algebraic geometry, Oxford Math. Monogr., The Clarendon Press, Oxford University Press, Oxford, 2006. http://dx.doi.org/10.1093/ acprof : oso/9780199296866.001.0001

Huy08 D. Huybrechts, Derived and abelian equivalence of K3 surfaces, J. Algebraic Geom. 17 (2008), no. $2,375-400$.

LM12 J. Lo and Y. More, Some examples of tilt-stable objects on threefolds, arXiv:1209.2749v1.

Mac96 A. Maciocia, Gieseker stability and the Fourier-Mukai transform for abelian surfaces, Quart. J. Math. Oxford Ser. (2) 47 (1996), no. 185, 87-100. http://dx.doi.org/10.1093/qmath/ 47.1 .87

Mac07 E. Macrì, Stability conditions on curves, Math. Res. Lett. 14 (2007) 657-672. http://dx. doi. org/10.4310/MRL.2007.v14.n4.a10

Mac12 E. Macrì, A generalized Bogomolov-Gieseker inequality for the three-dimensional projective space, Algebra Number Theory 8 (2014), no. 8, 173-190. http://dx.doi.org/10.2140/ant. 2014.8.173

Muk78 S. Mukai, Semi-homogeneous vector bundles on an Abelian variety, J. Math. Kyoto Univ. 18 (1978), no. 2, 239-272.

Muk81 S. Mukai, Duality between $D(X)$ and $D(\hat{X})$ with its application to Picard sheaves, Nagoya Math. J. 81 (1981), 153-175.

Oka06 S. Okada, Stability manifold of $\mathbb{P}^{1}$, J. Algebraic Geom. 15 (2006), no. 3, 487-505. http://dx. doi.org/10.1090/S1056-3911-06-00432-2

Orl02 D. Orlov, Derived categories of coherent sheaves on abelian varieties and equivalences between them, (Russian) Izv. Ross. Akad. Nauk Ser. Mat. 66 (2002), no. 3, 131-158; translation in Izv. Math. 66 (2002), no. 3, 569-594. http://dx.doi.org/10.1070/IM2002v066n03ABEH000389

Sch14 B. Schmidt, A generalized Bogomolov-Gieseker inequality for the smooth quadric threefold, Bull. Lond. Math. Soc. 46 (2014), no. 5, 915-923. http://dx.doi.org/10.1112/blms/bdu048

Sim92 C. Simpson, Higgs bundles and local systems, Publ. Math. Inst. Hautes Études Sci. 75 (1992), 5-95.

Tod14 Y. Toda, A note on Bogomolov-Gieseker type inequality for Calabi-Yau 3-folds, Proc. Amer. Math. Soc. 142 (2014), no. 10, 3387-3394. http://dx.doi.org/10.1090/ S0002-9939-2014-12096-X

Yos09 K. Yoshioka, Stability and the Fourier-Mukai transform II, Compos. Math. 145 (2009), no. 1, 112-142. http://dx.doi.org/10.1112/S0010437X08003758

Antony Maciocia A.Maciocia@ed.ac.uk

School of Mathematics, University of Edinburgh, The King's Buildings, Mayfield Road, Edinburgh, EH9 3JZ, United Kingdom

Dulip Piyaratne dulip.piyaratne@ipmu.jp

Kavli Institute for the Physics and Mathematics of the Universe, The University of Tokyo, 5-1-5

Kashiwanoha, Kashiwa, 277-8583, Japan 